\title{
Parallel and Population-specific Gene Regulatory Evolution in Cold-Adapted Fly Populations
}

Yuheng Huang ${ }^{1 * @}$, Justin B. Lack ${ }^{1 * *}$, Grant T. Hoppel ${ }^{1}$, and John E. Pool ${ }^{1 @}$

${ }^{1}$ Laboratory of Genetics, University of Wisconsin-Madison, Madison, Wisconsin 53706

*Current address: Department of Ecology and Evolutionary Biology, University of California, Irvine, Irvine, CA 92697

${ }^{* *}$ Current address: Advanced Biomedical Computational Science, Frederick National Laboratory for Cancer Research, Frederick, MD 21701

@e-mail: yuhenh3@uci.edu; jpool@wisc.edu. 


\section{Abstract}

2 Changes in gene regulation at multiple levels may comprise an important share of the molecular

3 changes underlying adaptive evolution in nature. However, few studies have assayed within- and

4 between-population variation in gene regulatory traits at a transcriptomic scale, and therefore

5 inferences about the characteristics of adaptive regulatory changes have been elusive. Here, we

6 assess quantitative trait differentiation in gene expression levels and alternative splicing (intron usage)

7 between three closely-related pairs of natural populations of Drosophila melanogaster from

8 contrasting thermal environments that reflect three separate instances of cold tolerance evolution.

9 The cold-adapted populations were known to show population genetic evidence for parallel evolution

10 at the SNP level, and here we find evidence for parallel expression evolution between them, with

11 stronger parallelism at larval and adult stages than for pupae. We also implement a flexible method to

12 estimate cis- versus trans-encoded contributions to expression or splicing differences at the adult

13 stage. The apparent contributions of cis-versus trans-regulation to adaptive evolution vary

14 substantially among population pairs. While two of three population pairs show a greater enrichment

15 of cis-regulatory differences among adaptation candidates, trans-regulatory differences are more

16 likely to be implicated in parallel expression changes between population pairs. Genes with

17 significant $c i s$-effects are enriched for signals of elevated genetic differentiation between cold- and

18 warm-adapted populations, suggesting that they are potential targets of local adaptation. These

19 findings expand our knowledge of adaptive gene regulatory evolution and our ability to make

20 inferences about this important and widespread process.

\section{Introduction}

23 Different species or populations often evolve similar phenotypes when adapting to similar

24 environments (Schluter 2000; Losos, 2011). Although such parallel phenotypic evolution can be

25 caused by amino acid changes, there is increasing evidence that regulatory mutations altering gene

26 expression underlie many cases of phenotypic evolution (Wittkopp \& Kalay, 2012; Jones et al. 2012;

27 Stern 2013; Sackton et al. 2019). Most studies on gene regulatory evolution focus on expression

28 abundance (the number of transcripts for a whole gene). However, alternative splicing changes

29 resulting in modified transcript proportions can also contribute to adaptation (Barbosa-Morais et al.

30 2012; Gamazon and Stranger 2014; Smith et al. 2018), and yet splicing evolution has received far

31 less study. 
33 The level of parallelism for gene expression evolution varies across study systems. In some taxa and

34 natural conditions, significantly more genes show parallel changes (repeatedly up- or down-regulated

35 in one ecotype relative to the other among independent population pairs) than anti-directional

36 changes (Zhao et al. 2015; Hart et al. 2018; Kitano et al. 2018; McGirr and Martin. 2018). However,

37 some other cases did not show significant parallel patterns, or they even showed anti-parallel patterns

38 (Derome et al. 2006; Lai et al. 2008; Hanson et al. 2017). The varying degree of parallelism may

39 partly be explained by the level of divergence among ancestors: more closely related ancestors are

40 expected to show a higher degree of parallel genetic evolution underlying similar phenotypic

41 evolution (Conte et al. 2012; Rosenblum et al. 2014).

43 Furthermore, gene expression evolution can be caused by the same or different molecular

44 underpinnings. Because of the difficulties of mapping expression quantitative trait loci (eQTLs), a

45 first step is to classify the expression evolution into two regulatory classes. Cis-regulatory changes

46 are caused by local regulatory mutations and result in allele-specific expression in a hybrid of

47 divergent parental lines (Singer-Sam et al. 1992; Cowles et al. 2002; Yan et al. 2002; Wittkopp et al.

48 2004). Trans-regulatory changes are caused by mutations at other loci. They modify the expression

49 of both alleles in hybrid diploids and do not result in allele-specific expression (Yvert et al. 2003;

50 Wittkopp et al. 2004; Wang et al. 2007). The relative importance of cis- and trans-effects to parallel

51 evolution varies among different studies systems (Wittkopp et al. 2008; McManus et al. 2010;

52 Wittkopp and Kalay 2012; Coolon et al. 2014; Lemmon et al. 2014; Nandamuri et al. 2018). Many

53 previous studies have focused on regulatory evolution between relatively distantly related lineages

54 such as different species, from which population genetic evidence of adaptive evolution may not be

55 available. Some studies have investigated the cis- vs. trans-regulatory variation within or between

56 recently diverged populations but are limited to one or two populations (Chen et al. 2015; Osada et al.

57 2017; Glaser-Schmitt et al. 2018). To our knowledge, the only two cases comparing cis- and trans-

58 regulatory changes for repeated adaptive divergence between populations are from threespine

59 stickleback fish and they revealed contrasting patterns (Hart et al. 2018; Verta and Jones 2019).

60 Hence, the relative contributions of $c i s$ - and trans-effects to recent parallel adaptation remain mostly

61 unknown.

62

63 In part driven by interest in the evolutionary response to climate change, Drosophila has been used as

64 a model system to study the genetic basis of thermal adaptation (Hoffmann et al. 2003). Because

65 temperature is an important environmental variable along latitudinal clines, clinal populations of 
Drosophila melanogaster have been studied for decades (Adrion et al. 2015). Along these clines, populations exhibit different degrees of cold tolerance in the expected direction, suggesting spatially varying selection related to temperature (Hoffmann and Weeks 2007; Schmidt and Paaby 2008). The recent development of genomics has allowed identification of clinal genomic variants, which are candidates for thermal adaptation (e.g., Kolaczkowski et al. 2011; Fabian et al. 2012; Bozicevic et al. 2016; Mateo et al. 2018). There is also evidence of parallel evolution at the genomic and transcriptomic level (Reinhardt et al. 2014; Bergland et al. 2015; Machado et al. 2015; Zhao et al. 2015; Juneja et al. 2016; Zhao and Begun 2017). Some of these studies compared clines between species (which may have somewhat distinct biology), while others compared clines between Australia and North America (which both feature primarily European ancestry with clinally variable African admixture). Other transcriptomic studies have identified genes showing differential expression between sub-Saharan African and European populations (e.g., Catalan et al. 2012; Huylmans and Parsch 2014), which are separated by moderately strong neutral genetic differentiation associated with the out-of-Africa bottleneck.

More broadly, populations of D. melanogaster from contrasting environments offer an excellent opportunity to study parallel gene regulatory evolution and its underlying mechanisms. Originating from a warm sub-Saharan ancestral range (Lachaise et al. 1988; Pool et al. 2012), D. melanogaster has occupied diverse habitats, including environments with contrasting temperature ranges. There are at least three instances in which the species expanded to cold environments: from Africa into higher latitude regions in Eurasia, from Ethiopia lowland to higher altitudes, and from South Africa lowland to higher altitudes. Populations were collected from these six regions, representing three warm-cold population pairs: Mediterranean pair (MED), collected in Egypt (EG, warm) and France (FR, cold); Ethiopian pair (ETH) collected in Ethiopia lowland (EA, warm) and highland (EF, cold); and South Africa pair (SAF), collected in South Africa lowland (SP, warm) and highland (SD, cold). Importantly, each of these population pairs has the advantage of low genetic differentiation between its warm- and cold-adapted members compared to the differentiations among pairs (Pool et al. 2017).

93 Although the cold populations have invaded colder habitats for only $\sim 1000-2000$ years $(\sim 15 \mathrm{k}-30 \mathrm{k}$

94 generations) (Sprengelmeyer et al. 2020) and different habitats have distinct selective pressures

95 besides cold (e.g., air pressure, ultraviolet radiation, food resources), the cold-dwelling populations

96 have shown signals of parallel adaptation for cold tolerance and allele frequency changes (Pool et al.

97 2017). In the present study, this unique system allows us to assess the degree of parallelism for

98 transcriptomic changes underlying parallel adaptation to colder environments. 
100 Because the selection environments can vary drastically across life stages of Drosophila, we may

101 expect to see different patterns of local adaptation and parallelism in gene expression across stages.

102 For D. melanogaster, the larvae are mostly located within fruit and their primary role is feeding. The

103 pupae are located on or near the fruit and are immobile. The adults are mobile; their primary role is

104 mating and reproduction (Powell, 1997; Sokolowski et al. 1986), and it is thought to be the primary

105 overwintering stage in seasonally cold environments (Lzquierdo 1991). And a recent study using $D$.

106 melanogaster populations across the globe found local adaptation to thermal environments at egg,

107 larval and adult stages but not the pupal stage (Austin and Moehring 2019). Therefore, we may

108 expect a different level of parallel gene expression evolution for thermal environments for the pupal

109 stage.

111 Here, we generate RNA sequencing (RNA-seq) data for multiple outbred genotypes from each of the

112 six population samples listed above, from larval, pupal, and adult stages. We estimate gene

113 expression and alternative intron usage levels for each sample, then identify cases of unusually high

114 quantitative trait differentiation between each pair of warm- and cold-adapted populations and

115 compare their genomic locations across developmental stages. We find that genes with highly

116 differentiated expression are enriched on the $\mathrm{X}$ chromosome in the adult stage relative to the larval

117 stage. We find evidence for parallel evolution for expression for both the larval and the female adult

118 stages, but less parallel signal for the pupal stage. We further tease out the cis- and trans-regulatory

119 effect at the adult stage by sequencing the transcriptomes of the parental lines from different

120 populations and their F1 offspring. Applying our resampling approach to study cis- and trans-

121 regulatory effects, we find that the relative contributions of these effects to adaptive expression

122 differentiation is quite variable across population pairs, with trans-effects showing greater

123 parallelism. Finally, we observe enrichments of genes with high $F_{S T}$ among those that showed cis-

124 effects and identify several candidate genes with both cis-effects and high $F_{S T}$, as potential targets of

125 local adaptation.

\section{Methods and Materials}

128 Ecologically and phenotypically differentiated populations

129 The three Drosophila melanogaster cold-warm population pairs used in this study, France-Egypt

130 (MED), Ethiopia (ETH) and South Africa (SAF), were described in previous publications (Pool et al. 
131 2012; Lack et al. 2015; Pool 2017). Previous study has shown that female adults from the cold

132 populations (FR, EF and SD) were more likely to recover after 96 hours at $4^{\circ} \mathrm{C}$ than the respective

133 warm populations (Pool et al. 2017). To extend these results, three inversion-free strains from each of

134 the cold populations as well as an ancestral warm adapted population (ZI) were used to measure egg

135 to adult viability at different temperatures. Viability was assayed at $15^{\circ} \mathrm{C}$ as the cold environment

136 and $25^{\circ} \mathrm{C}$ as the warm control environment. 40 mated female flies were allowed to lay eggs in a half

137 pint glass milk bottle with a standard medium at room temperature for 15 hours. Each strain occupied

$138 \sim 8$ bottles. After the flies were removed and the numbers of eggs were counted, about half of the

139 bottles were incubated at $25^{\circ} \mathrm{C}$ and the other half $15^{\circ} \mathrm{C}$. The numbers of adult flies that emerged from

140 each bottle were counted after 14 days and 42 days from warm and cold environments respectively.

141 Viability for each strain was measured as the average emergence proportion among bottles, which is

142 the number of emerged adults divided by the number of eggs. To determine significance, unpaired t-

143 tests between strains from each cold population and those from the ZI population were performed for

144 both temperature conditions.

RNA sample collection and sequencing

147 Within each population of the three warm/cold pairs (six populations in total), we selected 16 strains

148 and assigned them into eight crosses (Fig. 1). Before the crossing, all the strains had been inbred for

149 eight generations. The criterion for choosing parental strains for a cross was based on minimal

150 genomic regions of overlapping heterozygosity. Among the strains chosen within each population,

151 we used similar criteria to select four strains to perform crosses between the warm and the respective

152 cold populations. Two of the four strains were used as the maternal lines and the other two were used

153 as paternal lines in the between-population crosses. One cross between SD and SP populations was

154 lost. We also collected adult female samples from the parental inbred lines used in the between-

155 population crosses. Inversion frequencies are known to differ between these populations (Pool et al.

156 2017) and inversions have been associated with expression differences (Lavington \& Kern 2017;

157 Said et al. 2018). While inversions are not an explicit focus of our study, they may contribute to

158 population expression differences. The inversion information for the strains used can be found in

159 Table S1. 
A

162

163

164

165

166

167

168

169

170

171

172

173

174

175

176

177

178

179

180

181

182

183

184

185

186

187

B within-population cross

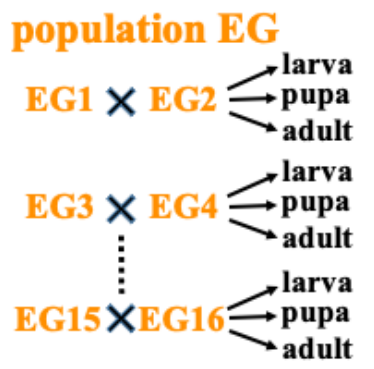

30

15

0
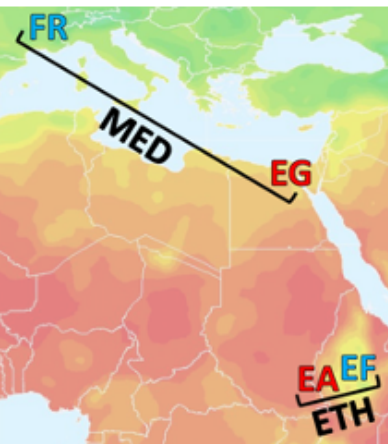

ZI

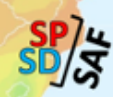

parental strain

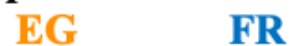

EG1 $\rightarrow$ adult $\quad$ FR1 $\rightarrow$ adult

EG2 $\rightarrow$ adult FR2 $\rightarrow$ adult

EG3 $\rightarrow$ adult FR3 $\rightarrow$ adult

EG4 $\rightarrow$ adult FR4 $\rightarrow$ adult

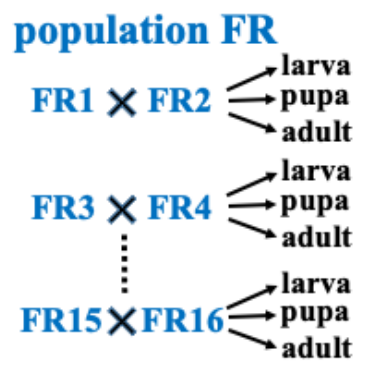

between-population cross

EG FR

EG1 X FR1 $\rightarrow$ adult

EG2 $\times$ FR2 $\rightarrow$ adult

EG3 $\times$ FR3 $\rightarrow$ adult

EG4 $\times$ FR4 $\rightarrow$ adult

Fig 1. Illustrations of the geographic origins of the three population pairs and the crossing design. (A) A map of average year-round temperature $\left({ }^{\circ} \mathrm{C}\right)$ showing the geographic origins of each population sample studied, and their groupings into pairs of closely-related warm- and cold-derived population samples. The Mediterranean (MED) pair comprises a cold-derived France population (FR) and a warm-derived Egypt population (EG). The Ethiopian (ETH) pair comprises a cold-derived highaltitude population (EF) and a warm-derived low-altitude population (EA). Likewise, the South African (SAF) pair comprises a cold-derived high-altitude population (SD) and a warm-derived lowaltitude population (SP). The location of an additional warm-derived population from Zambia (ZI), within the species' putative ancestral range, is also indicated. (B) Schematic figure showing the crossing design for one population pair (MED) as an example. Within-population crosses generated controlled outbred offspring for estimating $P_{S T}$ to quantify population differentiation in gene expression; samples at three developmental stages (third instar larva, pupa and female adult) were collected from each cross. Parental inbred strains from warm- and cold-adapted populations and inter-population crosses between them were studied to estimate cis- and trans-regulatory effects that underlie the expression divergence; samples from female adults were collected.

All the flies were reared at $15^{\circ} \mathrm{C}$, which approximated the derived cold condition. 20 virgin females and 20 males were collected from maternal and paternal lines respectively for each cross and allowed to mate and lay eggs for a week in half-pint bottles. Each bottle contained standard Drosophila medium (containing molasses, cornmeal, yeast, agar, and antimicrobial agents). For the withinpopulation crosses, samples at three developmental stages were collected: larva, pupa and female adult. Third-instar larvae were collected on the surface of the medium. For pupa, new yellow pupae were collected within one day of pupation. For adult, female flies were collected 4-5 days after eclosion. For samples from between-population crosses and parental lines, only female adults were collected. All the samples were shock-frozen in liquid nitrogen immediately after collection. 
189 Approximate 50 larvae or 50 pupae or 30 female adults were used for RNA extraction for each

190 sample. Total mRNA was extracted using the Magnetic mRNA Isolation Kit (New England Biolabs,

191 Ipswich, MA) and RNeasy MinElute Cleanup Kit (Qiagen, Hilden, Germany). Strand-specific

192 libraries were prepared using the NEBNext mRNA Library Prep Reagent Set for Illumina. Libraries

193 were size-selected for approximately 150 bp inserts using AMPureXP beads (Beckman Coulter, CA,

194 USA). The 179 libraries were quantified using Bioanalyzer and manually multiplexed for

195 sequencing. All libraries were sequenced on a HiSeq2500 (V4) with 75bp paired-end reads in two

196 flow cells. Numbers of paired-end reads generated for each library can be found in Table S2.

198 Quantifying gene expression and exon usage frequency

199 The paired-end sequence reads for the within-population cross samples were mapped to the

200 transcribed regions annotated in the D. melanogaster reference genome (release 6, BDGP6.84) using

201 STAR with parameters from ENCODE3's STAR-RSEM pipeline (Li and Dewey 2011; Dobin et al.

202 2013). We note that cold- and warm-derived members of each population pair are expected to have

203 very similar genome-wide reference sequence divergence (Lack et al. 2016a). For gene expression,

204 the numbers of reads mapped to each gene were quantified using RSEM (Li and Dewey 2011). Reads

205 mapped to the rRNA were excluded in the analysis. The expression abundance for each gene was

206 standardized by the numbers of reads mapped to the total transcriptome of the sample.

208 To quantify exon usage, we used Leafcutter (Li et al. 2018) to estimate the excision frequencies of

209 alternative introns. This phenotype summarizes different major splicing events, including skipped

210 exons, and 5' and 3' alternative splice-site usage. Leafcutter took the alignment files generated by

211 STAR as input to quantify the usage of each intron. Then Leafcutter formed clusters that contain all

212 overlapping introns that shared a donor or accept splice site. The default parameters were used: $\geq 50$

213 reads supporting each intron cluster and $\leq 500 \mathrm{~kb}$ for introns length. The exon usage frequency is the

214 number of intron excision events divided by the total events per cluster. It is worth noting that

215 Leafcutter only detects exon-exon junction usage and it is unable to quantify 5' and 3' end usage and

216 intron retention (Alasoo et al. 2019), which were not examined here.

217

218 Principal component analysis 
219 To visually assess the overall patterns of variation in the transcriptomes among samples, we first

220 performed principal component analysis (PCA) for the within population cross samples across three

221 developmental stages using DESeq2 (Love et al. 2014). The DESeq dataset object was constructed

222 from the matrix of the count data outputted from RSEM. After the variance stabilizing transformation

223 (vst), the top 5000 genes with highest variance across samples at the transformed scale were used for

224 PCA. The principal component value for each sample was obtained by the function plotPCA (Fig.

225 S2). We also performed principle component analysis for samples at each developmental stage. For

226 the adult stage, we included the F1 offspring from crosses within populations, F1 offspring from

227 crosses between populations and the inbred parental lines of the latter crosses.

Identifying outliers in gene expression and intron usage differentiation using within-population

230 crosses

231 To identify candidate genes under differential evolution between the warm and cold populations in

232 each pair, we first controlled for the potential transcriptome skew caused by very highly expressed

233 genes. For each expressed gene, we calculated the average expression of the cold samples

$234\left(A v g \operatorname{Exp}_{\text {cold }}\right)$ and that of the warm samples $\left(A v g \operatorname{Exp}_{\text {warm }}\right)$. Then we obtained the median of the ratio of

$235 A v g \operatorname{Exp}_{\text {cold }} / A v g \operatorname{Exp}_{\text {warm }}$ across all expressed genes for the population pair. Gene expression for the

236 warm samples was normalized by multiplying this median before subsequent analysis. This

237 correction was designed to avoid a scenario in which either the cold population or the warm

238 population had important expression changes in one or more highly expressed genes that caused the

239 relative expression of all other genes to shift, even if their absolute expression level did not.

We used $P_{S T}$ statistics to quantify gene expression divergence between cold and warm populations in each population pair using samples from within-population crosses:

$$
P_{S T}=\frac{V_{\text {between }}}{V_{\text {between }}+2 V_{\text {within }}}
$$

243 where $V_{\text {between }}$ is between-populations variance for expression abundance and $V_{\text {within }}$ is the average

244 variance for expression abundance within populations. Although both within- and between-

245 population components of variance can be confounded by the environmental variance, $P_{S T}$ is still a

246 useful statistic to quantify phenotypic differentiation (Lande 1992; Spitze 1993; Merila 1997;

247 Brommer 2011; Leinonen et al. 2013). Here, environmental variance should be reduced by the

248 common laboratory environment. To reduce sampling variance before calculating $P_{S T}$, for each gene,

249 we required the total mapped reads across all 48 within-population samples to exceed 200 for a given 
developmental stage. Then for each population/stage, we excluded the crosses/samples with the highest and lowest gene expression for each gene (to avoid high $P_{S T}$ values being driven by single anomalous values), resulting in six samples per population/stage. The $P_{S T}$ quantile based on data excluding extreme samples is concordant with the $P_{S T}$ quantile calculated using all the crosses for most cases (Fig. S3).

We chose the above $P_{S T}$-based approach instead of simply testing for differential expression in part because our within-population samples reflect real variation as opposed to technical replicates. Also, many alternative methods make assumptions about the data (e.g., negative binomial distribution for transcript counts) which are difficult to apply to splicing, even if they hold for expression. $P_{S T}$ and the population genetic index $F_{S T}$ are under the same theoretical framework, and are often directly compared to search for evidence of adaptive trait differentiation. However, environmental and measurement variance will downwardly bias $P_{S T}$, making targets of local adaptation less likely to reach a threshold defined by genome-wide high $F_{S T}$ outliers. Hence, in this study we simply focus on the highest quantiles of $P_{S T}$ for a given trait/population comparison, as detailed below.

As with gene expression, we used $P_{S T}$ to estimate the intron usage differentiation between cold and warm populations, with $V_{\text {between }}$ as the between-population variance for a given intron's usage frequency, $V_{\text {within }}$ as the average within-population variance for intron usage frequency. Before calculating $P_{S T}$, for each exon-exon junction, we averaged the intron excision events $\left(n_{i}\right)$ and the alternative events $\left(n_{j}\right)$ of the cluster across all samples in a developmental stage. The minimum for

271 both types of event had to be at least $5\left(n \in\left[n_{i}, n_{j}\right] \geq 5\right)$. We also required that at least six samples

272 have intron usage count $>0$ in each population for the exon-exon junction to be included in

273 subsequent analysis. Then for each exon-exon junction, we excluded the sample with highest and

274 lowest intron usage in a population/stage and calculated $P_{S T}$.

276 Examining the relative contribution of the $X$ chromosome to population differentiation across

277 stages

278 For gene expression differentiation, we used the upper 5\% quantile of $P_{S T}$ as outlier cutoff to identify

279 candidate genes potentially under geographically differential selection. Then we calculated the

280 fraction of the outliers located on the $\mathrm{X}$ chromosome $\left(f_{x}\right)$. To generate the null distribution of $f_{x}$, we

281 permuted the genes used in calculating $P_{S T}$ and calculated $f_{x}$, for the top 5\% of the permuted gene set. 
This process was repeated 10,000 times to obtain a null distribution of $f_{x}$. The upper $2.5 \%$ and lower $97.5 \%$ quantile of $f_{x}$, define the $95 \%$ confidence interval. To test whether the actual $f_{x}$ is significantly different from the null, the $\mathrm{p}$-value is calculated as two times the proportion of the $f_{x}$ ' that were equal or more extreme than the actual $f_{x}$ (two-tailed test).

To test whether the developmental stage impacts the enrichment of outliers on the $\mathrm{X}$ chromosome, we analyzed the fraction of genes on the $\mathrm{X}$ chromosome (f) using linear model (lm function) in $\mathrm{R}$ (version 3.6.3):

where Stage is larvae/pupal/adult. Type is outliers or nonoutliers. Pair is the population pair. As we are interested in whether the difference in $f$ between outliers and the background depends on the

293 development stage, the model above was compared to a reduced model without the interaction term

294 Stage $\times$ Type using likelihood ratio test (anova function with test $=$ "LRT"). If the Stage $\times$ Type for

295 the full dataset was significant, we performed the same analysis separately for larva-pupa, pupa-adult and larva-adult datasets to determine which stages caused the significant Stage $\times$ Type effect.

\section{Comparing $P_{S T}$ outliers with published data for African and European populations}

299 To study whether the adult fly in MED pair changes expression in similar ways as other African and

300 European population comparisons, we first obtained lists of candidate genes showing significant

301 differential expression between African and European populations for adult samples from Muller et

302 al. 2011 (padj < 0.05) and von Hecket et al. 2016. We calculated the numbers of $P_{S T}$ outliers that are

303 shared with the published lists with between-populations differential gene expression (in the same 304 directions of expression changes). Then we permuted all the genes we tested in the MED pair and 305 selected the same number of genes as the true outliers randomly. We asked how many genes in the 306 randomly permuted list are shared with the published lists. We repeated the process 10,000 times to 307 obtain a null distribution of the shared numbers. The p-value was calculated as the proportion of the 308 null distribution that was equal or more than the actual number of shared genes (one-tailed test).

309 Another test was whether the shared genes for $P_{S T}$ outliers were more likely to change expression co310 directionally with the published lists than the non-outliers, which was tested by a Chi-square test. 
313 To study the degree of parallel evolution in gene expression, we identified outlier genes shared

314 between two population pairs and showing consistent changes in the cold populations relative to the

315 warm ones (co-directional). Whether the number of shared outliers with co-directional change was

316 significantly different from expected by chance was determined by a permutation-based test. For the

317 outlier genes in a certain pair, we calculated the number of these genes $(N)$ that were shared and

318 changed expression in the same direction in the outliers from another pair. To generate the null

319 distribution of $N$, we permuted the genes used in calculating $P_{S T}$ and obtained a set of genes that pass

320 a certain quantile in each pair. Then the $N$ ' was calculated based on the two permuted sets of outliers.

321 This process was repeated 10,000 times to obtain a null distribution of $N$ '. To determine the

322 statistical significance, a p-value was calculated as two times the proportion of the $N$ ' that were equal

323 or more extreme than the actual $N$ (two-tailed test). The statistics here and those below assume the

324 expression changes are independent among genes/introns, which is not always the case (genes can

325 interact with each other via regulatory networks). We performed similar tests for pairwise

326 comparisons between developmental stages for each population pair. The numbers for shared outliers

327 with consistent changes between pairwise stages were reported in Table S8.

329 The second approach used to examine parallelism of gene expression evolution was to focus on the

330 outlier genes for a specific population pair and examined whether the expression changes in another

331 pair followed the same directions. If cold adaptation causes similar evolution in gene expression,

332 those genes should tend to show changes in the same directions in both pairs. Each of the pairwise

333 population combinations had two comparisons: the outliers can come from either pair. For the outlier

334 genes in a certain pair, we calculated the fraction $(F)$ of these genes changing expression in the same

335 direction in another pair. To generate the null distribution of $F$, we permuted the genes used in

336 calculating $P_{S T}$ and calculated $F$ ' for the permuted genes that pass a certain quantile. This process

337 was repeated 10,000 times to obtain a null distribution of $F^{\prime}$. The upper $2.5 \%$ and lower $97.5 \%$

338 quantile of the distribution define the $95 \%$ confidence interval. The p-value was calculated as two

339 times the proportion of the $F^{\prime}$ that were equal or more extreme than the actual $F$ (two-tailed test).

341 To identify intron usage outliers, a cutoff of the upper 5\% $P_{S T}$ was used. If multiple exon junctions

342 had $P_{S T}$ above the top 5\% cutoff, only the exon junction with the highest $P_{S T}$ would be kept as an

343 outlier to control for nonindependence. Because the numbers of shared intron usage outliers in both

344 population pairs are small $(<10)$, we only performed the second type of analysis described above. For

345 a certain developmental stage, we used the top 5\% outlier intron usage in a particular pair and asked 
346 what percentages of the intron usage changed co-directionally in another pair. To determine the

347 statistical significance, we used the permutation approach as described above.

GO enrichment test for $P_{S T}$ outlier genes

350 The Gene Ontology enrichment tests were performed using the R package "clusterProfiler" (Yu et al. 2012) based on the fly genome annotation (Carlson 2018). The types of GO terms being tested

352 contained all three sub-ontologies: Biological Process (BP), Cellular Component (CC) and Molecular

353 Function (MF). Selection of overrepresented GO terms was based on adjusted p-value $<0.1$ using

354 the "BH" method (Benjamini and Hochberg 1995) for each sub-ontology. This relaxed p-value

355 threshold (after accounting for multiple testing) was used in light of the hypothesis-generating goals

356 of this analysis. For gene expression, the upper $5 \% P_{S T}$ outliers were tested for GO enrichment

357 relative to all the expressed genes for each population pair for a certain stage. To determine whether

358 the shared significant GO terms between pairs were more than expected by chance, we randomly

359 sampled the same numbers of genes as the outliers and performed the GO test for both pairs and

360 identified the shared significant GO terms between pairs. We repeated the process 1000 times to get a

361 set of numbers for the shared significant GO terms and compared to the actual number of shared

362 significant GO terms to get a permuted p-value.

364 To access the functional categories of the differential intron usage, we calculated the quantile of $P_{S T}$

365 for each alternative intron's usage. To rank the differentiation for a gene, we used the highest

366 quantile (the most extreme differentiation) among the intron usages within the gene as the gene

367 quantile $\left(q_{g e n e}\right)$. To account for the multiple testing of the intron usages for a gene, the adjusted total

368 numbers of testing is calculated as $n_{\text {sum }}=\sum_{i=1}^{i=j}\left(n_{i}-1\right)$, where $n_{i}$ is the number of testing for a

369 cluster and $j$ is the number of clusters for the gene. Then, the adjusted gene quantile is $q^{\prime}$ gene $=1$ - $(1-$

$\left.370 q_{\text {gene }}\right) \mathrm{x} n_{\text {sum }}$. The upper $5 \% q_{\text {gene }}$ was used to identify the most differentiated genes for intron usage

371 and they were tested for GO enrichment as described above.

373 Estimating cis-and trans-effects of regulatory divergence using between-population crosses and

\section{4 parental strains}

375 To study the contributions of cis- and trans-regulatory effects on expression and intron usage

376 divergence, we focused our analysis on the upper $5 \% P_{S T}$ outliers for gene expression/intron usage.

377 For each gene/intron junction in each population pair, we selected a representative cross showing the 
greatest difference between parental strains for this analysis. In addition, this difference needed to be larger than the average difference between the cold and warm populations from the outbred crosses

380 for its pair.

To study allele-specific expression/intron junction usage, we obtained the genomic sequences of the two parental strains aligned separately to the FlyBase D. melanogaster 5.77 assembly (Lack et al. 2015; 2016a). The SNP calling from the reference genome was done by samtools (Li et al. 2009). To avoid mapping bias for the RNAseq reads (Degner et al. 2009; Stevenson et al. 2013), we updated the reference based on the SNPs for the two parental stains by masking the SNPs as "N". The F1 female adult RNA-seq reads were mapped to the updated reference using STAR with options: -chimFilter None --outFilterMultimapNmax 1 (Dobin et al. 2013). Because of the fairly high level of heterozygosity within our inbred lines (Lack et al. 2015), we attempted to use polymorphic sites to study the allele-specific expression instead of focusing on the fixed difference between parental strains. However, based on the simulations we performed (see supplementary document), our new method requires a large number of $F 1$ offspring ( $>300$ per cross) to reduce the random sampling of parental alleles. For this experiment (only 30 F1 offspring per cross), we therefore used SNPs that were fixed differences between the parental strains. SNPs were filtered with read counts $\geq 10$ in the F1 RNA-seq sample and the parental samples. Then the allele frequency in the RNA reads for the F1 sample was calculated to estimate allelic expression proportion. The allelic expression proportion for each candidate gene $p_{F I}$ was the median average allele frequency for all sites located in the gene region.

We tested two null hypotheses corresponding to cis-only and trans-only regulatory differences using a resampling approach. Under the null hypothesis that cis-regulatory effects are absent, the $p_{F 1}$ is expected to be near 0.5 because the cold parental strain contributes half of the alleles to F1 offspring, and alleles from different parents are expressed similarly in these F1s (Cowles et al. 2002; McManus 2010; Meiklejohn et al. 2014). Under the null hypothesis that trans-regulatory effects are absent, $p_{F 1}$ is expected to approximate the ratio of the cold parental strain expression to the total expression of both parental strains (Wittkopp et al. 2004): $r_{F 0}=E_{c} /\left(E_{c}+E_{w}\right)$. However, sampling effects can cause 
411 until we reached the numbers of reads mapped to the gene. Then we recalculated the $p_{F 1}$ ' for each

412 SNP and then averaged across sites for each gene. We repeated the above process 1000 times to get a

413 distribution of $p_{F 1}$ '. A 95\% confidence interval of the distribution not overlapping with 0.5 suggested

414 the existence of a cis-effect.

416 To test for a trans-effect, the uncertainty when estimating the expression level in parental strains also

417 needs to be accounted for. For each gene/intron in a parental strain, we used binomial sampling based

418 on the expression level of the gene/intron. The sampling probability is the proportion of reads for that

419 gene/intron relative to total reads in a sample and the number of sampling events equals the total

420 reads of the sample. Then we had the updated expression for the cold strain $E_{c}$ ' and the warm strain

$421 E_{w}{ }^{\prime}$. The updated $r_{F 0}$ ' is calculated as $E_{c}{ }^{\prime} /\left(E_{c}{ }^{\prime}+E_{w}{ }^{\prime}\right)$. The sampling and calculation were repeated

4221000 times. Each time the $r_{F 0}$ ' was paired with a $p_{F 1}$ ' described above to calculate the difference $D^{\prime}=$

$423 r_{F 0}{ }^{\prime}-p_{F 1}$ '. A $95 \%$ confidence interval of $D$ ' not overlapping with 0 suggested the existence of a

424 trans-effect.

426 To test the specificity and sensitivity of this approach, we performed simulations to generate

427 expression read data and apply our method on the simulated data (supplementary document). We

428 found that our approach has good performance under reasonable conditions and can be adapted for

429 other traits, such as splicing. For splicing, the $p_{F 1}$ is the allele frequency for the diagnostic SNPs

430 located in the exon-junction and the $r_{F 0}$ is the ratio of the cold parental strain intron usage frequency

431 to the sum of the frequencies for both parental strains.

433 Based on the tests above, the set of candidate genes were classified into categories including no

434 significant cis- or trans-effect, cis only, and trans only (McManus 2010; Schaefke et al. 2013; Chen

435 et al. 2015). For genes showing both cis- and trans-effects, we further classified them based on

436 whether these two effects favored expression of the same (co-directional) or different parental allele

437 (anti-directional). For exon usage differentiation, we applied a similar approach to classify the

438 differentiated exons into the five categories, accounting for different sampling effects and

439 measurement errors. Instead of analyzing expression level of the parental strains $(E)$, we analyzed

440 their intron usage frequency for the sets of outlier intron junctions. 
443 To study the level of co-regulation among outliers related to cold adaptation, we focused on the

444 expressions of the outliers in the cold-derived populations. For each pairwise combination of two

445 outliers, we calculated the correlation coefficient of the expression values among the eight outbred

446 samples. To test whether the correlation coefficient is different from random expectation, we

447 permuted the eight outbred samples randomly for one gene for each gene combination, requiring at

448 least five of the eight samples to be changed. Then we calculated the correlation coefficient between

449 genes with the permuted samples. We repeated the process 10,000 times to obtain a null distribution

450 of the correlation coefficient. The p-value was calculated as the proportion of the null distribution

451 that was equal or more than the actual coefficient (one-tailed test). We used $p<0.05$ as a cutoff to

452 identify significantly co-regulated outlier pairs. To compare the level of co-regulation between

453 populations, we calculated the proportion of co-regulated pairs were significantly co-regulated for

454 each population as well as the number of significant co-regulated partners for each gene in each

455 population.

\section{Examining between-population genetic differentiation for genes with cis-effect}

458 For the $P_{S T}$ outliers identified with significant $c i s$-effects, we hypothesized that causative $c i s$ -

459 regulatory elements may show elevated allele frequency differentiation between the warm and cold

460 populations. For expression abundance, the majority of cis-regulatory SNPs are located within $2 \mathrm{~kb}$

461 upstream of the transcription start site and downstream of the transcription end site (Massouras et al.

462 2012). Therefore, we used the interval from $2 \mathrm{~kb}$ upstream to $2 \mathrm{~kb}$ downstream as the focal region of a

463 gene for this analysis. We calculated window $F_{S T}$ and SNP $F_{S T}$ using sequenced genomes from

464 Drosophila Genome Nexus (Lack et al. 2015 \& 2016a). For window $F_{S T}$, the division of windows

465 within a gene region was based on 250 non-singleton variable sites per window in the ZI population

466 (Pool et al. 2017). Each window needed to have at least five genotypes for each population. Before

467 assigning window $F_{S T}$ to the focal genes, we confirmed that there is no large chromosomal scale of

468 differentiation between populations for each pair (Fig. S8). The highest $F_{S T}$ for the windows

469 overlapping the focal region was assigned as its $F_{S T_{-} w i n m a x}$. To determinate the statistical significance

470 of $F_{S T_{-} \text {winmax }}$, we calculated $F_{S T_{-} \text {winmax }}$ for all other blocks of the same number of windows (to account

471 for gene length) along the same chromosome arm where cross-over rates were above $0.5 \mathrm{cM} / \mathrm{Mb}$

472 (Comeron et al. 2012), but excluding those within 10 windows of the focal region. The specific non-

473 low recombination regions are: $2.3-21.4 \square \mathrm{Mb}$ for the $\mathrm{X}$ chromosome, $0.5-17.5 \square \mathrm{Mb}$ for arm $2 \mathrm{~L}, 5.2-$

$47420.8 \square \mathrm{Mb}$ for arm 2R, 0.6-17.7 $\square \mathrm{Mb}$ for arm 3L, and 6.9-26.6 $\square \mathrm{Mb}$ for arm 3R. SNP $F_{S T}$ was

475 calculated for sites with at least 10 alleles for each population. The highest value $\left(F_{S T_{-} S N P \max }\right)$ within 
476 the focal region was thus obtained for the focal gene. Analogous to our $F_{S T_{-} \text {winmax }}$ permutation, we

477 also calculated $F_{S T \_S N P m a x}$ for permuted regions with the same number of SNPs as the focal region,

478 along the non-low cross-over rate region on the same chromosome arm. For both $F_{S T_{-} \text {winmax }}$ and

$479 F_{S T_{-} S N P \max }$, we then focused on regions in the upper $5 \%$ quantile of permuted values for further

480 analysis. To test whether the expression outliers with cis-effects are enriched for high $F_{S T}$ outliers, we

481 analyzed the fraction of $F_{S T}$ outliers $(f)$ using a linear model $(\mathrm{lm})$ in R:

$$
f=\text { Type }+ \text { Pair }
$$

483 where Type is outliers with cis-effect (cis-outlier) or non-outliers, and Pair is the population pair. The

484 model above was compared to a reduced model without the Type term using likelihood ratio test

485 (anova function with test = "LRT"). For outlier genes that showed a cis-effect and high $F_{S T}$, location

486 and functional information were obtained from Flybase (Thurmond et al. 2019).

\section{Results}

\section{Gene expression differentiations between warm-and cold-derived populations}

491 In a cold environment $\left(15^{\circ} \mathrm{C}\right)$, we found the FR and $\mathrm{EF}$ populations have significantly higher egg-to-

492 adult viability than an ancestral range population and the SD population follows the same trend (Fig.

$493 \mathrm{~S} 1$, Table S3). In contrast, at a $25^{\circ} \mathrm{C}$ benign temperature all of the populations have relatively high

494 survival (75\%). These findings were consistent with past results (Pool et al. 2017) in suggesting that

495 the cold-derived populations have adapted to low temperature.

497 We then surveyed the transcriptomes of larvae, pupae, and female adults for multiple genotypes from 498 each cold- and warm-adapted population using high-throughput RNA sequencing (RNA-Seq). To

499 focus on the transcriptomes of outbred genotypes, we generated eight within-population crosses from 500 each population under the derived cold environment $\left(15^{\circ} \mathrm{C}\right)$. We pursued this outbred strategy to 501 guard against inbreeding effects amplifying within-population regulatory variation and therefore

502 dampening estimates of regulatory differentiation between populations (estimated using the 503 quantitative genetic index $\left.P_{S T}\right)$. Comparing our inbred adult RNA-Seq data against outbred data from 504 a subset of the same strains, we found results that were mostly consistent with that expectation: five 505 out of six populations showed greater expression variance in inbred than outbred data, and two out of 506 three population pairs showed higher $P_{S T}$ values from outbred than inbred data (Table S6). We 507 therefore focused on outbred data for subsequent population comparisons. 
509 After performing PCA on normalized expression values, we found that the first and second principle

510 component of gene expression showed clear signals of developmental stages among samples, while

511 signals of transcriptome-wide population differentiation was more modest (Fig. S2). We then used

$512 P_{S T}$ to quantify phenotypic differentiation of expression and splicing between populations in each

513 pair. $P_{S T}$, analogous to $F_{S T}$ for genetic variation, measures the amount of trait variance between

514 populations versus total variance for a phenotype (Merila 1997; Brommer 2011; Leinonen et al.

515 2013). The genes/intron usages with highest $P_{S T}$ quantiles are more likely to be under ecological

516 differential selection between populations than those with lower $P_{S T}$ quantiles (Leder et al. 2015).

518 Genes were filtered for analysis based on $\geq 200$ counts across all 48 within-population samples (8

519 samples per population, six populations in total for three pairs). The numbers of genes that passed the

520 filters for analysis were: 4699 genes for larva, 5098 genes for pupa and 6785 genes for adult. We

521 initially observed that in the ETH pair, the adult sample showed a general shift in transcriptome-wide

522 relative abundances between populations, caused by a few highly expressed genes (Fig. S4). Further

523 investigation suggested that the EF population was primarily responsible for the observed ETH

524 asymmetries in adults (Fig. S5). Many of the highly expressed genes in the cold-adapted and larger-

525 bodied EF population (Lack et al. 2016b) are related to muscle protein (Table S4). To correct for the

526 influence of such changes on relative expression levels, we standardized the expression values of

527 warm-derived populations by the median expression ratio between cold- and warm-derived

528 populations, resulting in about the same numbers of genes with increased and decreased expression

529 in the cold-derived population relative to the warm-derived one transcriptome-wide (Fig. S4). To

530 study gene expression divergence potentially under ecologically differential selection, we calculated

$531 P_{S T}$ (Materials and Methods). The $P_{S T}$ values for all genes for each population/stage are listed in

532 Table S4. We used the upper 5\% quantile of $P_{S T}$ as outliers for each population pair. For the outliers,

533 there is a strong directionality on the expression difference between populations for the ETH pair

534 (Fig. 2): a large majority of ETH $P_{S T}$ outliers had higher expression in the cold-adapted EF

535 population in larvae and especially adults, with pupae showing a reversed pattern. These asymmetries

536 mirrored transcriptome-wide skews in expression proportion for this population pair, in that

537 substantially more genes had higher EF expression than the modal proportion (Fig S4). These

538 observations suggest unique regulatory features for the populations in the ETH pair, perhaps hinting

539 that many outlier genes might be co-regulated. 

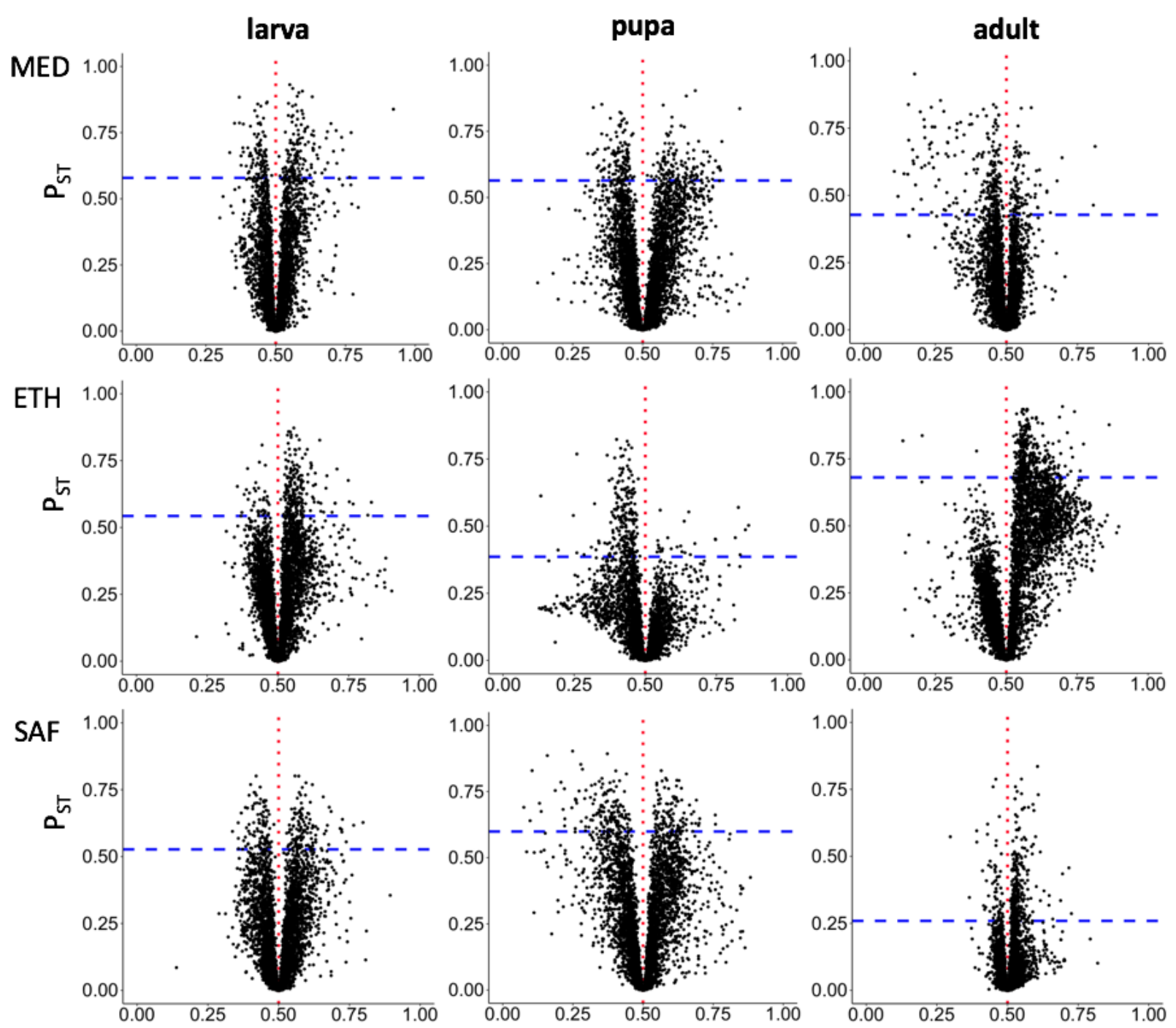

540

Cold-derived Population Expr. Prop.

Figure 2. The relationship between expression differences between populations and $P_{S T}$ illustrates asymmetric expression differences in some population pairs / developmental stages. The $\mathrm{x}$-axis is the cold-derived population expression proportion, which is the ratio of mean expression of the coldderived population relative to the sum of mean expression values from the two populations. Proportion higher than 0.5 (red vertical dashed lines) indicate a higher expression for the coldderived population and the warm-derived one. The blue horizontal dashed lines show the upper 5\% quantile of $P_{S T}$.

\section{Comparing $P_{\text {ST }}$ Outliers with published studies}

Since there are studies comparing whole-body transcriptomes between African and European populations in this species (Muller et al. 2011; von Heckel et al. 2016), we examined whether our $P_{S T}$ outliers for adults from the MED pair overlapped with the candidates showing differential expression between African and European populations from the published datasets. While each of these analyses 
555 might detect regulatory evolution that occurred in Europe, we emphasize the distinctness of these

556 geographic comparisons, since our Egypt sample differs substantially from the Zimbabwe population

557 featured in those studies. For upper 5\% quantile of $P_{S T}$ outliers, we do not see more sharing with the

558 previous candidates from either study than random expectations (permutation test, $\mathrm{p}=0.47$ for

559 comparing with Muller et al. 2011 and $\mathrm{p}=0.39$ for comparing with von Heckel et al. 2016).

560 However, for the upper $10 \%$ of $P_{S T}$, the outliers of MED are more likely to be shared with the

561 candidates from Muller et al. 2011 than random expectations (permutation test, $\mathrm{p}=0.0033$ ) but not

562 with those from von Heckel et al. 2016 (permutation test, $\mathrm{p}=0.47$ ). Moreover, among all the shared

563 genes, our outliers were more likely to show gene expression change in the same directions as the

564 previous candidates than the non-outliers $\left(\chi^{2}=6.3, \mathrm{df}=1, \mathrm{p}=0.012\right.$ for comparing with Muller et al.

$5652011, \chi^{2}=7.4, \mathrm{df}=1, \mathrm{p}=0.0065$ for comparing with von Heckel et al. 2016).

\section{$X$ chromosomal and autosomal contributions to regulatory evolution}

568 To investigate the contribution of autosomes versus the $\mathrm{X}$ chromosome to the expression

569 differentiation due to cold adaptation, we surveyed the locations of $P_{S T}$ outliers for different

570 developmental stages (Fig. 3). At the larval stage, the proportions of $P_{S T}$ outliers located on the $\mathrm{X}$

571 chromosome were lower than the genome-wide level in each population pair (permutation test, $\mathrm{p}=$

5720.72 for MED; $p=0.0076$ for ETH; $p=0.021$ for SAF). In contrast, at the adult (female) stage, the

573 proportions of outliers located on the $\mathrm{X}$ tended to be higher than the background level (permutation

574 test, $\mathrm{p}=0.30$ for MED; $\mathrm{p}=0.013$ for ETH and $\mathrm{p}=0.89$ for $\mathrm{SAF}$ ). For pupa, the $\mathrm{X}$ chromosome

575 enrichment was not significantly different between outliers and the background. Considering the

576 three population pairs together, the relative enrichments of outliers on the $\mathrm{X}$ chromosome were

577 significantly different among developmental stages (likelihood ratio test, $\mathrm{p}=0.048$ ). The patterns of

578 different enrichment among stages were caused by larva/adult differences (likelihood ratio test, $\mathrm{p}=$

5790.0058 for larva-adult; $\mathrm{p}=0.25$ for pupa-adult; $\mathrm{p}=0.24$ for larva-pupa). The larva/adult difference

580 might suggest either (1) a greater abundance of genes affecting adult fitness on the X chromosome,

581 as previously suggested (Gibson et al. 2001; Innocenti and Morrow 2010), (2) a greater influence of

582 female fitness on X chromosome evolution (e.g. Vicoso \& Charlesworth 2006), in light of sex

583 differences between our adult (female) and larval (mixed sex) samples, or (3) differences in the

584 contributions of local adaptation and genetic drift to outlier sets at different stages, in combination

585 with differential effects of drift between the X chromosome and autosomes (e.g. Pool \& Nielsen

586 2007). 

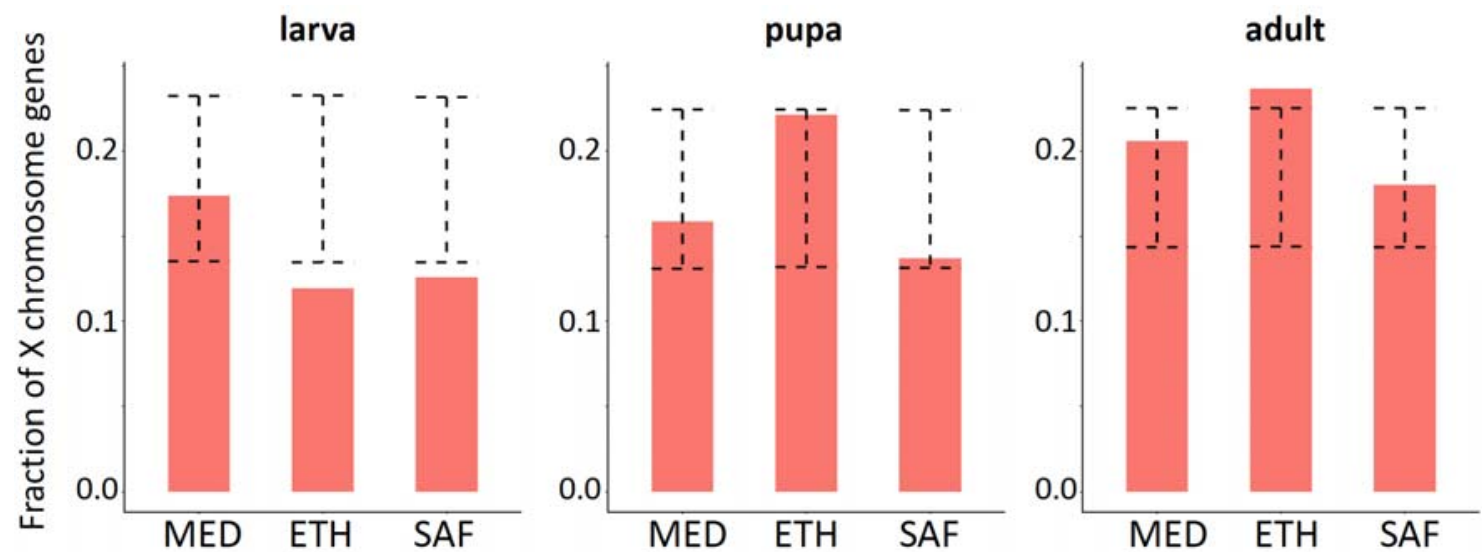

588 Fig 3. The fraction of $P_{S T}$ outliers located on the $\mathrm{X}$ chromosome varies between developmental stages.

589 The dashed error bar indicates the $95 \%$ confidence interval for the permuted data. If the real data 590 (orange bar) is outside the range of the error bar, it indicates the fraction is significantly different from the genomic background $(\mathrm{p}<0.05)$.

\section{Co-directional evolution in gene expression between population pairs}

Among the upper 5\% of $P_{S T}$ outliers, we found at least some significant signals of parallel expression divergence in all three pairwise comparisons (MED vs. ETH; MED vs. SAF; ETH vs. SAF), where the shared outliers with co-directional changes (i.e., expression difference between cold- and warmderived populations in the same direction for both pairs) were more abundant than expected by random permutation (Table 1; Fig 4A). Averaged across three pairwise comparisons, $6.7 \%$ of the outliers were shared and changed consistently for the adult stage while $4.7 \%$ of outliers were codirectional for larva and only $2.6 \%$ were co-directional for pupa (for which one population pair comparison for pupa showed significant anti-directional changes). Changing the $P_{S T}$ outlier cutoffs to $2.5 \%$ or $10 \%$ produced qualitatively similar patterns (Table S7). We found one shared outlier with co-directional changes among all three pairs for the adult stage: $I m l 1$, a regulator of cell size, starvation response, TOR signalling, and meiosis initiation (Bar-Peled et al. 2013; Wei et al. 2014). No three-pair shared outliers were found for the larva and pupa stages. One shared outlier among three pairs is not significantly more than the expectation by permutation. Still, it is worth noting that changing the $P_{S T}$ outlier cutoff to $10 \%$ results in 12 shared outliers with co-directional changes among three pairs (permutation test, $\mathrm{p}<0.0001$ ), which suggests that meaningful regulatory evolution may extend beyond our defined outliers. The names, $P_{S T}$ values and quantiles of these 12 genes are shown in Table 2. Of these genes, we note the role of CrebB in circadian behavior, which

612 et al. 2015; Cao \& Edery 2017; Helfrich-Förster et al. 2018). Overall, these analyses suggest that the 613 adult stage has the highest level of parallel evolution while pupa has the lowest. 


\begin{tabular}{|l|l|l|l|l|l|l|}
\hline stage & larva & pupa & adult & \\
\hline $\begin{array}{l}\text { Population } \\
\text { pairs }\end{array}$ & MED & SAF & MED & SAF & MED & SAF \\
\hline ETH & $3.4 \%$ & $3.0 \%$ & $\mathbf{5 . 5 \%}$ & $2.0 \%$ & $3.0 \%$ & $\mathbf{1 0 . 1 \%}$ \\
& $(3.0 \%)$ & $(3.4 \%)$ & $(2.7 \%)$ & $(2.0 \%)$ & $(2.4 \%)$ & $(2.9 \%)$ \\
\hline SAF & $\mathbf{7 . 7 \%}$ & & $\mathbf{0 . 4 \%}$ & & $\mathbf{5 . 3 \%}$ & \\
& $(3.0 \%)$ & & $(2.0 \%)$ & & $(2.4 \%)$ & \\
\hline
\end{tabular}

Table 1. Evidence for parallel expression evolution between population pairs. The percentages of $P_{S T}$ outlier with parallel expression abundance changes are shown. The random expectation is the median of the permuted proportions (in brackets). The majority of proportions were higher than the expectation, with larvae and adult stages showing stronger patterns than the pupa. Those that were significantly different from the random expectation are in bold (permutation test, $\mathrm{p}<0.01$ ). We found one case, the MED-SAF comparison at pupal stage, that showed a significantly lower level of parallel evolution than the random expectation. Further detail regarding the numbers of shared and non-shared outliers can be found in Figure 4A.

\begin{tabular}{|l|l|l|l|l|l|l|}
\hline Pair & \multicolumn{4}{|l}{ MED } & \multicolumn{4}{l}{ ETH } & SAF \\
\hline Gene name & $P_{S T}$ value & quantile & $P_{S T}$ value & quantile & $P_{S T}$ value & quantile \\
\hline Iml1 & 0.58 & 0.0177 & 0.85 & 0.0055 & 0.79 & 0.0001 \\
\hline larp & 0.42 & 0.0532 & 0.70 & 0.0401 & 0.51 & 0.0074 \\
\hline Smox & 0.49 & 0.0345 & 0.66 & 0.0607 & 0.49 & 0.0087 \\
\hline sky & 0.38 & 0.0694 & 0.68 & 0.0529 & 0.47 & 0.0102 \\
\hline AGO1 & 0.36 & 0.0818 & 0.70 & 0.0423 & 0.45 & 0.0134 \\
\hline CG10365 & 0.53 & 0.0251 & 0.67 & 0.0553 & 0.39 & 0.0195 \\
\hline Nepl3 & 0.35 & 0.0840 & 0.64 & 0.0744 & 0.34 & 0.0270 \\
\hline CG42674 & 0.34 & 0.0948 & 0.84 & 0.0057 & 0.29 & 0.0383 \\
\hline CrebB & 0.44 & 0.0461 & 0.74 & 0.0268 & 0.24 & 0.0566 \\
\hline Cka & 0.56 & 0.0202 & 0.75 & 0.0249 & 0.23 & 0.0606 \\
\hline par-1 & 0.34 & 0.0883 & 0.70 & 0.0396 & 0.22 & 0.0713 \\
\hline CG5116 & 0.45 & 0.0432 & 0.78 & 0.0171 & 0.20 & 0.0796 \\
\hline
\end{tabular}

Table 2. The $P_{S T}$ values and quantiles for the 12 genes that passed the top $10 \%$ cutoff with consistent expression changes across three population pairs at the adult stage.

627 The analysis above requires genes being outliers in both population pairs, which is quite restrictive 628 (the naïve expected proportion of sharing between two pairs under a $5 \% P_{S T}$ cutoff is $5 \% \times 5 \% \times 0.5=$ 
$6290.125 \%$ ) and may miss some broader patterns of parallel changes. We therefore performed a

630 complementary analysis which only required genes being outliers in one population pair and

631 examined whether the expression for this set of genes changed in the same direction in another pair,

632 regardless of outlier status in the latter pair. For example, 235 genes were outliers in the MED pair at

633 the larval stage. Then in another pair, we calculated the fraction of the 235 genes with expression

634 differences between cold- and warm-derived populations in the same direction as for MED. There

635 were excesses of co-directional changes for the larval stage (Figure 4B). The patterns were weaker

636 for the adult stage and there were excesses of anti-directional changes for the pupal stage. Changing

637 the $P_{S T}$ cutoff to $2.5 \%$ or $10 \%$ produce qualitatively similar patterns (Fig. S6).

638

A
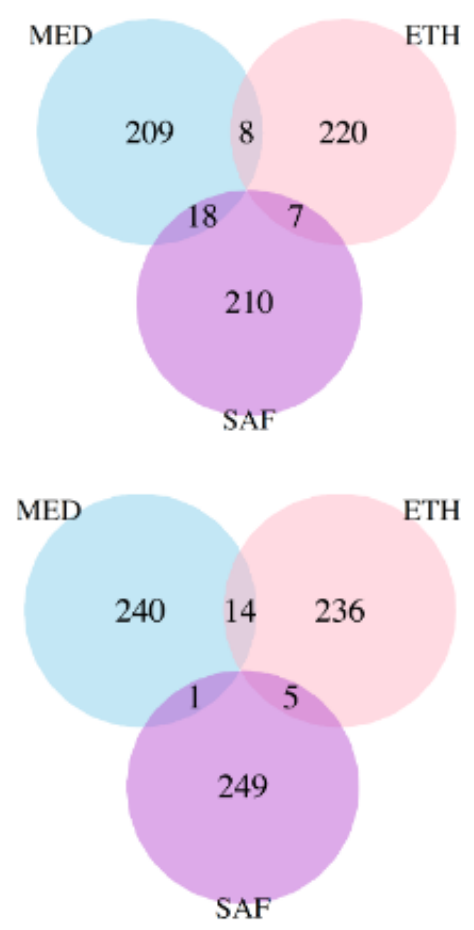

MED

ETH

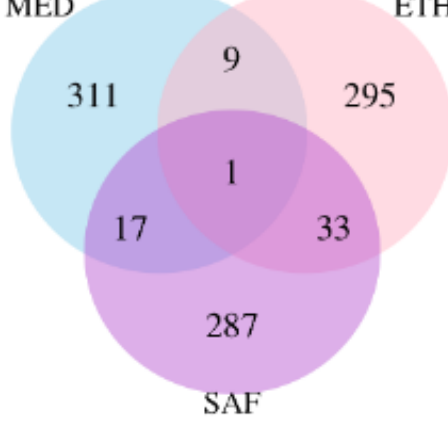

639

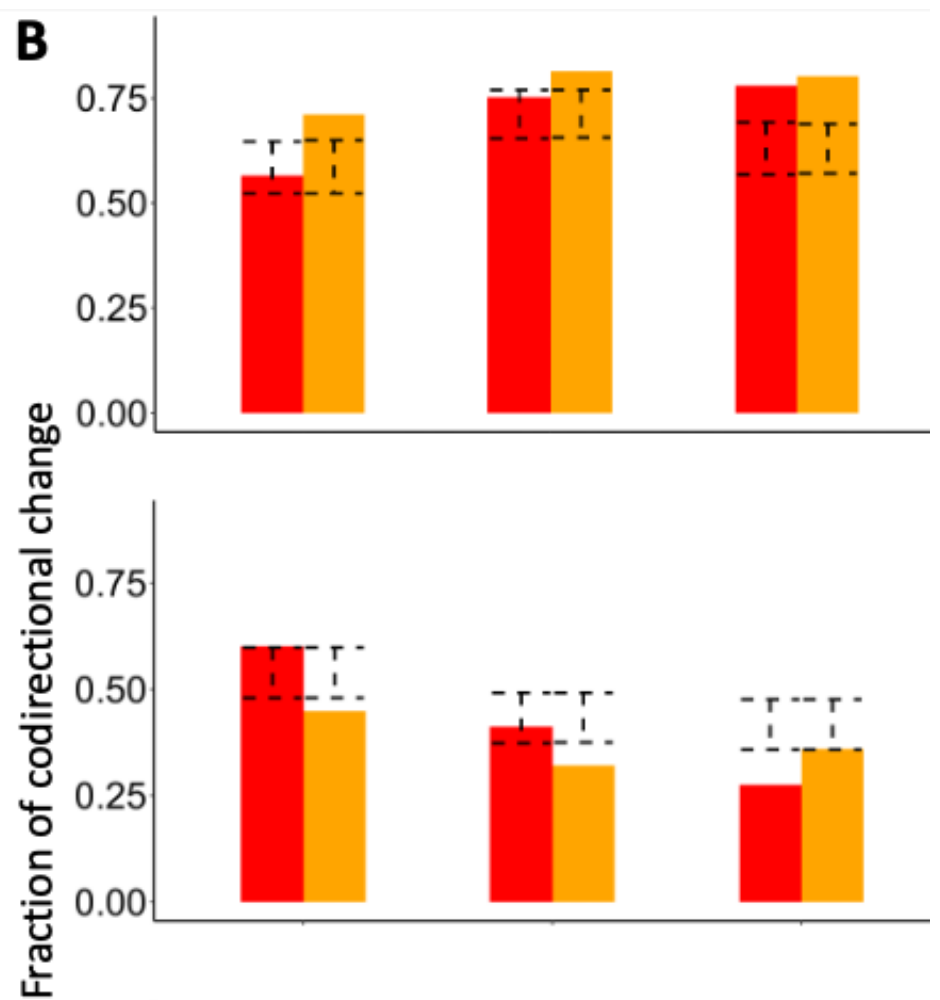

pupa

adult

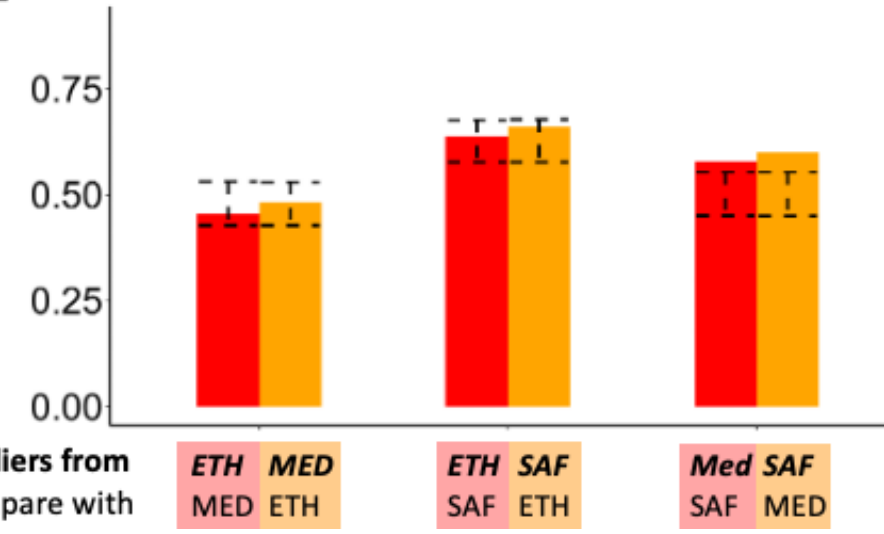


Fig. 4. Extent and direction of parallel evolution in gene expression among population pairs. (A) Venn diagrams for the numbers of shared outliers with co-directional changes between pairs and the rest of genes. (B) Fractions of co-directional gene expression changes between pairs for the $P_{S T}$ outliers identified in one pair of them. The name above is the pair used to identify outliers. The name below is the other pair in the comparison. The outliers used for each bar is the summed number of the pair in the Venn diagram. The dashed error bar indicates the $95 \%$ confidence interval for the fraction of co-directional expression changes in permuted data. If the real data is outside the range of the error bar, it indicates the fraction is significantly different from random expectation $(\mathrm{p}<0.05$, two-sided test based on permuted distribution).

We also performed similar analyses for $P_{S T}$ outliers of alternative intron usage. The numbers of intron-excision junctions that passed the cutoffs for $P_{S T}$ calculation were 4520 for larva, 5574 for pupa, and 7367 for adult. The adjusted gene quantiles for splicing are listed in Table S5. The patterns of co-directional changes were qualitatively similar to those for gene expression (Fig. S7). The fractions of co-directional changes were still highest for the larvae among the three stages; all of the comparisons except one showed an excess of co-directional changes relative to the background level, although only one comparison is significant based on the permutation test (outliers from MED being co-directionally expressed in SAF). Overall, the patterns for co-directional changes were weaker for splicing than those for gene expression. Table S9 lists the genes with the top $20 P_{S T}$ for both expression and intron usage for each population pair. An interesting example is curled, which is an extreme splicing outlier for ETH and MED, and an extreme expression outlier for ETH as well. Also known as nocturnin, one isoform of this gene is thought to have a dedicated role in Enriched functional categories for the $P_{S T}$ outliers

665 Significant Gene Ontology (GO) terms enriched in different sets of $P_{S T}$ outliers for gene expression

666 are listed in Table S10. Among the significant GO terms for different population pairs, we found five 667 terms shared between the MED pair (24 significant terms) and ETH pair (47 significant terms) at the 668 adult stage (mitochondrion, nucleoside metabolic process, ribonucleoside metabolic process, purine 669 nucleoside metabolic process and oxidation-reduction process). The level of sharing was

670 significantly more than expected by chance based on permuted outlier sets ( $p<0.001$, no shared GO

671 terms were found in the permuted datasets), suggesting functional convergence for adult

672 development to the cold environment for the MED pair and ETH pair. Further, similar (though non-

673 identical) GO terms were identified from different pairs at different stages such as terms related to

674 mitochondria, nucleoside metabolism, and oxidoreductase complex. However, the majority of GO 
675 terms were unique for different pairs, suggesting that many functional changes for adaptation to cold

676 environments may be population-specific. For intron usage, we only found one significant GO term

677 for SAF pair at the larval stage (meiotic cell cycle).

Cis- and trans-acting contributions to differential gene expression at the adult stage

680 Gene expression differentiation can be caused by cis- or trans-regulatory effects. A cis-effect comes

681 from a local regulatory mutation and results in an allele-specific expression difference in a F1 hybrid;

682 while a trans-effect is caused by remote loci that modify both alleles in a diploid cell. Therefore, a

683 cis-effect can be estimated by the allelic expression in F1 hybrid (Cowles et al. 2002). We quantify

684 that effect based on the expression proportion in the F1 offspring of a between-population cross for

685 the allele from the cold population minus 0.5 (null expectation when $c$ is-effect absent). A trans-effect

686 can be estimated by the expression difference between parents that was not attributed to the cis-effect

687 (Wittkopp et al. 2004), as described in the Materials and Methods.

689 First, we described the transcriptome-wide patterns of cis-and trans-effect sizes across all analyzed 690 genes at the adult stage. The magnitudes of trans-effect sizes were significantly larger than the cis-

691 effect sizes in all three population pairs (mean absolute cis-effects and trans-effects were: MED pair, 6920.07 vs. 0.16, p < 2.2e-16; ETH pair, 0.07 vs. 0.16, p < 2.2e-16; SAF pair, 0.09 vs. 0.11, p < 2.2e-16.

693 'Mann-Whitney' paired test.). Moreover, we found strong negative relationships between cis- and

694 trans-effects within each population pair (Fig. S7), where the cis- and trans-effects were generally in

695 the opposite directions. Although the pattern can be biologically meaningful, it may also represent an

696 artifact from using the same F1 expression data for allele specific expression (ASE) estimation to

697 infer both cis- and trans-effects. Any measurement error on ASE will introduce an artifactual

698 negative correlation between cis- and trans-acting changes (see Discussion below).

700 Next, we used our flexible permutation approach (see Materials and Methods and supplementary 701 document) to study how many genes show a significant cis-effect, trans-effect, or both among the 702 three population pairs (Table 3). Fig. 5A shows a graphical depiction of cis- and trans-effects on F1 703 and parental samples, illustrating cis-effects influence both F1 allele-specific expression and the 704 parental expression ratio while trans-effects only influence the parental expression ratio. Averaged 705 across population pairs, about $60 \%$ of genes show significant cis-and/or trans-effects (62\% for 706 outliers and $59 \%$ for non-outliers). We also found that $19 \%$ of genes show cis-regulatory effects 
707 while $53 \%$ of them show trans-effects, consistent with trans-effects being stronger on average than

708 cis-effects. This apparently greater prevalence of trans-regulatory evolution was observed in spite of 709 our lesser power to detect trans- relative to cis-effects (Supplementary Text).

710

\begin{tabular}{|c|c|c|c|c|c|c|c|}
\hline pair & $\begin{array}{c}\text { expression } \\
\text { type }\end{array}$ & $\begin{array}{c}\text { Total } \\
\text { tests }\end{array}$ & Cis only & Trans only & $\begin{array}{c}\text { Both co- } \\
\text { dir }\end{array}$ & $\begin{array}{c}\text { Both anti- } \\
\text { dir }\end{array}$ & Neither \\
\hline MED & $\begin{array}{c}P_{S T} \\
\text { outliers }\end{array}$ & 184 & $20(11 \%)$ & $74(40 \%)$ & $6(3 \%)$ & $14(8 \%)$ & $70(38 \%)$ \\
\hline $\begin{array}{c}\text { Non- } \\
\text { outliers }\end{array}$ & 4182 & $199(5 \%)$ & $2203(53 \%)$ & $99(2 \%)$ & $324(8 \%)$ & $1357(32 \%)$ \\
\hline $\begin{array}{c}P_{S T} \\
\text { outliers }\end{array}$ & 231 & $12(5 \%)$ & $136(59 \%)$ & $10(4 \%)$ & $14(6 \%)$ & $59(26 \%)$ \\
\hline $\begin{array}{c}\text { Non- } \\
\text { outliers }\end{array}$ & 4569 & $297(7 \%)$ & $2224(49 \%)$ & $137(3 \%)$ & $379(8 \%)$ & $1532(34 \%)$ \\
\hline SAF & $\begin{array}{c}P_{S T} \\
\text { outliers } \\
\text { Non- } \\
\text { outliers }\end{array}$ & 3993 & $250(6 \%)$ & $691(17 \%)$ & $40(1 \%)$ & $738(18 \%)$ & $2274(57 \%)$ \\
\hline
\end{tabular}

711 Table 3. The relative prevalence of significant cis- and trans-regulatory differences for $P_{S T}$ outliers

712 versus non-outliers varies among population pairs. Numbers of gene expression abundance traits

713 showing different regulatory effects for $P_{S T}$ outliers and non-outliers are shown. The percentage in

714 parentheses indicates the fraction of genes in each category relative to total genes in the tests.

715 

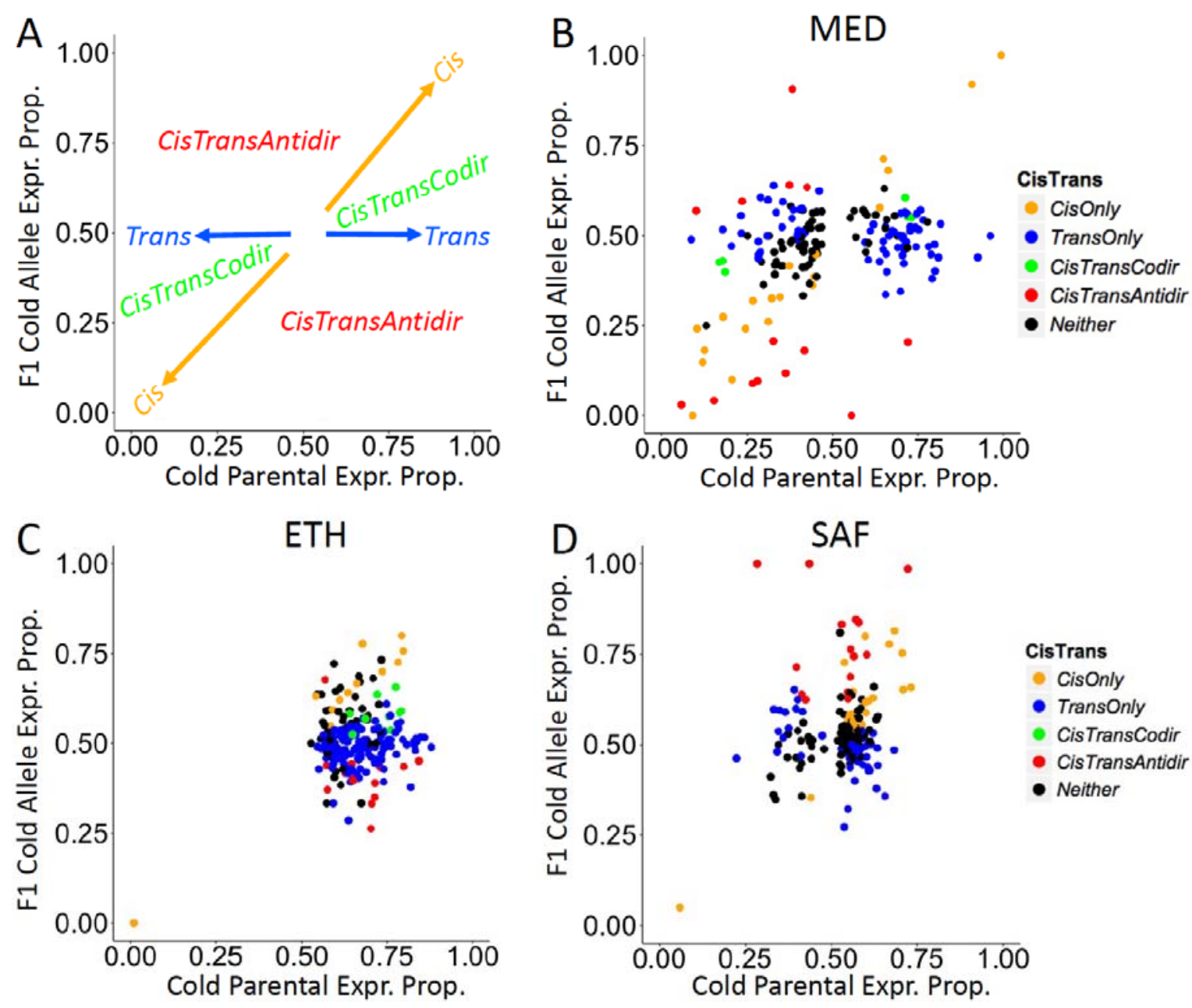

Fig 5. Population pairs show distinct patterns of $c i s$ - and trans-regulatory evolution for expression outliers. These plots depict evidence for cis- and trans-regulatory evolution based on the relative expression proportion of cold strain alleles in parental and F1 datasets. (A) Conceptual depiction of cis- and trans-effects. Cis-effects change the F1 allele-specific expression and the parental expression proportion concordantly (along the one-to-one ratio line). Trans-effects only change the parental expression proportion but not the $\mathrm{F} 1$ allele-specific expression (vertical line $\mathrm{y}=0.5$ ). Co-directional cis- and trans-effects (CisTransCodir) locate in the two spaces within the $45^{\circ}$ angle between the Cis vector and the Trans vector. Anti-directional cis- and trans-effects (CisTransAntidir) locate in the two spaces within $135^{\circ}$ angle between $C i$ is vector and Trans vector. (B-D) Evidence for $c i s$ - and trans-regulatory evolution of putatively adaptive expression differences between warm- and cold-

729 To examine the cis- and trans-regulatory contributions to adaptive evolution of gene expression, we 730 compared genes showing cis- or trans-effects between $P_{S T}$ outliers (Fig. 5B-D) and non-outliers.

731 Because of the potential artifact generating opposing cis-and trans-effects, we excluded genes

732 showing both significant cis- and trans-effects in opposite directions (Both anti-dir category in Table

733 3). For genes showing significant cis-effects (Fig. 6A), they were enriched in the outliers relative to 
734 the non-outliers for the MED pair $\left(\chi^{2}=11.6, \mathrm{df}=1, \mathrm{p}=0.00066\right)$ and the SAF pair $\left(\chi^{2}=4.8, \mathrm{df}=1\right.$,

$735 \mathrm{p}=0.029)$ but not for the ETH pair $(\chi 2<0.01, \mathrm{df}=1, \mathrm{p}=1)$. While for significant trans-effects

736 genes (Fig. 6B), they were enriched in the outliers relative to the non-outliers for the ETH pair $\left(\chi^{2}=\right.$

$7379.6, \mathrm{df}=1, \mathrm{p}=0.0019)$ and the SAF pair $(\chi 2=5.4, \mathrm{df}=1, \mathrm{p}=0.020)$ but the enrichment is opposite

738 for the MED pair $(\chi 2=10.2, \mathrm{df}=1, \mathrm{p}=0.0014)$.

740 Because theory suggests that co-regulation of genes can amplify the contribution of trans-effects (Liu

741 et al. 2019), we examined the level of co-regulation between pairwise outliers by calculating the

742 correlation coefficient between the expression values among the eight outbred samples within each

743 cold-derived population. Indeed, the percentage of pairwise correlation with evidence of co-

744 regulation (p-value < 0.05) is much higher in EF population than that in FR and SD populations (Fig.

745 6C, Table S11). At the gene level, the median number of significant co-regulatory partners for EF is

746 about twofold that for FR and SD (Fig. 6D). This pattern supports the hypothesis that substantial co-

747 regulation of outlier genes results in more significant trans-effects for the ETH pair (Fig. 6B).

748 Further, the strong co-regulation in EF might be related to the asymmetry observed in the ETH

749 outliers (Fig. 2). However, the pattern could reflect variation in cell type content among strains rather

750 than co-regulation within cells (see Discussion). 

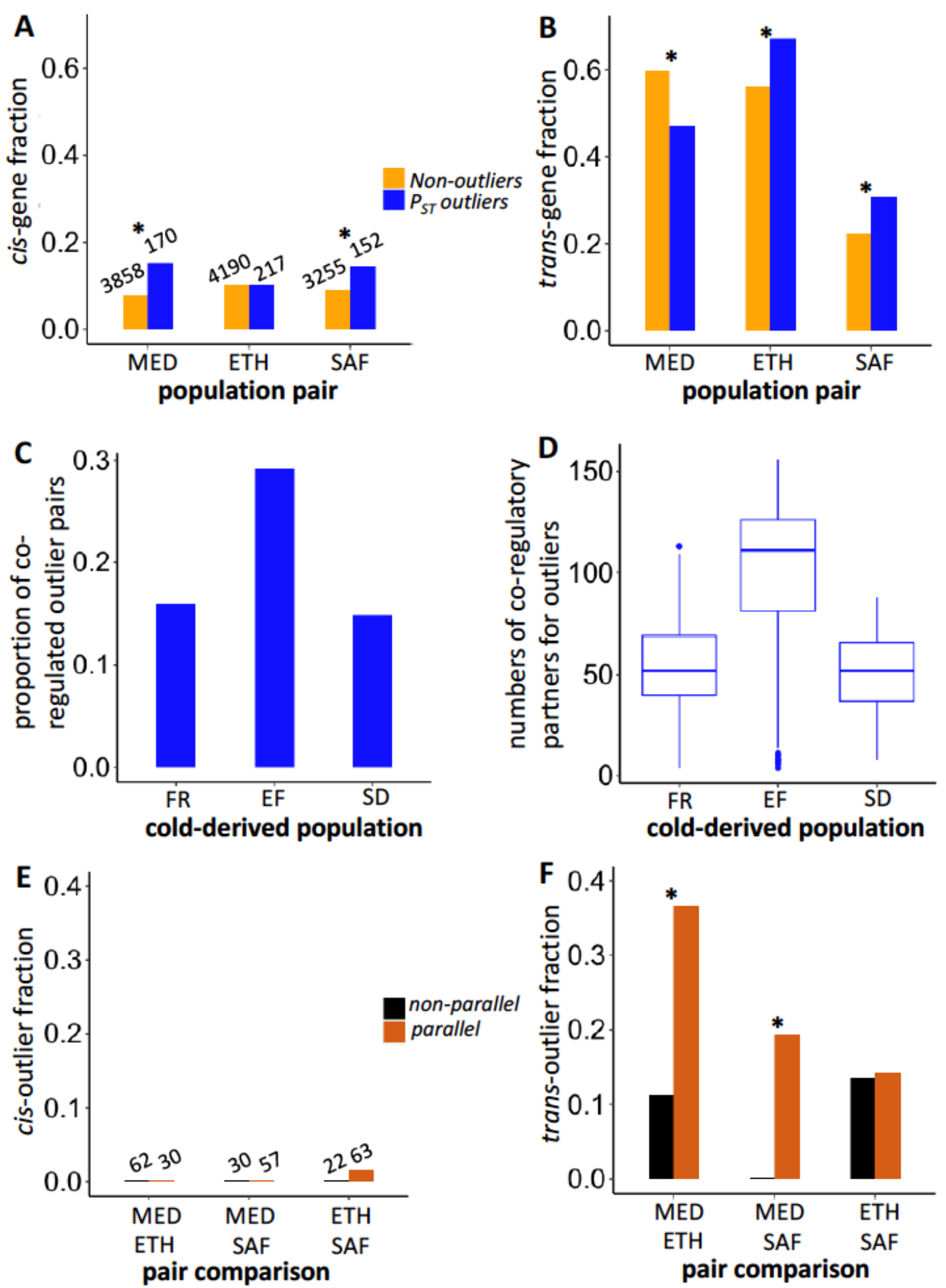

Fig 6. The prevalence of $c i s$ - versus trans-regulatory $P_{S T}$ outliers differs between population pairs, while trans changes show greater parallelism. The upper panels show the fractions of genes with cis regulatory effects (A) and those with trans-effects (B) for $P_{S T}$ outliers and non-outliers in each population pair. The middle panels show the proportion of co-regulated pairs among all pairwise outliers (C) and the numbers of co-regulatory partners for outlier genes (D) in each cold-derived 
population. The lower panels show the fractions of parallel outlier genes (i.e. shared and codirectional between population pairs) and non-paralleled outlier genes (i.e. shared and antidirectional between population pairs) showing cis-effect $(\mathrm{E})$ and trans-effect $(\mathrm{F})$ in different population pairs. The number above the bar for the cis-effect shows the denominator of the fraction, which applies to the trans-effect results as well. * indicates the fractions are significantly different between two categories $(\mathrm{p}<0.05)$.

To study the role of cis- and trans-regulatory effects on the parallel adaptation, we further focused on the outlier genes. We categorized the top $10 \% P_{S T}$ outliers based on whether they were shared between two pairs with consistent expression changes (parallel) or opposite changes (non-parallel). Because genes with cis- (or trans-) effects in both population pairs in the parallel category would indicate the contribution of cis- (or trans-) effects to parallel adaptive evolution, we identified shared outlier genes with cis- (or trans-) effects in any two pairs in both parallel and non-parallel categories (excluding genes showing both significant $c i s$ - and trans-effects in opposite directions). For ciseffects, only one gene (CG42788) is significant in both ETH and SAF pairs (Fig. 6E). While for trans-effects, the numbers of genes showing trans-effect are four for the MED and ETH pairs (CG8034, CG33981, kst and $w d b$ ), two for the MED and SAF pairs ( $s c f$ and $A P-2 \sigma$ ) and four for the ETH and SAF pairs (CG7766, Dlg5, larp and Pink1). Of these, larp had $P_{S T}$ quantiles near or below 0.05 in all three population pairs (Table 2); its functions include mitochondrial regulation (Zhang et al. 2019). There are significant enrichments of trans-effect genes in the parallel outlier category relative to the non-parallel category in two out of three population pair comparisons (Fig. 6F, MED \& ETH: $\mathrm{p}=0.0097$; MED \& SAF: $\mathrm{p}=0.014$; ETH \& SAF: $\mathrm{p}=1$; Fisher's Exact Tests). As a complementary analysis, we also included the non-shared outliers in the non-parallel category, and we found qualitatively similar patterns for cis- and trans-effects (Fig. S9). Overall, there is stronger evidence of trans-regulatory evolution contributing to parallel gene expression changes between

782 cold-adapted populations.

Cis- and trans-acting contributions to differential intron usage at the adult stage

785 For all intron usage traits, we found the magnitude of trans-effects on average to be higher than that 786 of cis-effects (mean absolute cis-effects and trans-effects are: MED pair, 0.13 vs. 0.17, p = 0.0001;

787 ETH pair, 0.30 vs. 0.32, p = 0.04; SAF pair, 0.17 vs. 0.19, $\mathrm{p}=0.0044$. 'Mann-Whitney' paired test).

788 Because of the limited diagnostic SNPs with enough read depth located in the intron junction regions, 789 there are few outlier introns tested for cis- and trans-regulatory effects (Table S12). 
Elevated genetic differentiation at cis-regulated expression outliers

792 Since the cis-regulatory mutations contributing to local adaptation may show differentiation in allele

793 frequency between populations, we examined genetic differentiation for expression outliers with cis-

794 effects (including cis-only genes and genes with both cis- and trans-effects to increase sample sizes).

795 We examined whether each of these cis-outliers shows high $F_{S T}$ between that pair of cold- and warm-

796 adapted populations - for both window $F_{S T}\left(F_{S T_{-} \text {winmax }}\right)$ and maximum SNP $F_{S T}\left(F_{S T_{-} S N P \max }\right)$. A gene

797 showing both significant $c i s$-effect and higher $F_{S T}$ quantile (here, the top 5\% versus comparable

798 genomic regions) could reflect adaptive regulatory evolution targeting the surveyed sequences or

799 nearby sites. We first confirmed that there is little chromosomal scale differentiation between

800 populations (aside from known moderate X-autosome differences for the MED pair; Lack et al. 2015)

801 by plotting window $F_{S T}$ across the major chromosome arms (Fig. S8). We observed no obvious

802 clustering of $P_{S T}$ outliers along the chromosome arms. For window $F_{S T}$ (Fig. 7A), high $F_{S T}$ is

803 enriched in cis-effect outliers relative to the non-outliers for the MED pair $(\mathrm{p}=0.022$. Fisher's Exact

804 Test) and the other two pairs show the same trend (ETH: $p=0.22$; SAF: $p=0.23$ ). Although the

805 MED result would be only marginally significant if correcting for the three tests performed, a clearer

806 result is obtained when considering the three population pairs together. In this analysis, the fractions

807 of genes with high window $F_{S T}$ were significantly higher in cis-outliers than that in non-outliers

808 (likelihood ratio test, $\mathrm{p}=0.0056$ ). For maximum SNP $F_{S T}($ Fig. 7B), the MED and SAF pairs showed

809 significant enrichments of high $F_{S T}$ in cis-effect outliers versus the non-outliers, while the ETH pair

810 showed a weak trend in that direction (MED: $\mathrm{p}=0.012 ; \mathrm{ETH}: \mathrm{p}=0.49 ; \mathrm{SAF}: \mathrm{p}=0.030$ ). Similar

811 analysis combining the three pairs found that the fractions of high maximum SNP $F_{S T}$ were

812 significantly higher in cis-outliers than that in non-outliers (likelihood ratio test, $\mathrm{p}=0.0045$ ). These

813 results reflected more than two-fold enrichment of $F_{S T}$ outliers among cis-regulated $P_{S T}$ outliers

814 across population pairs.

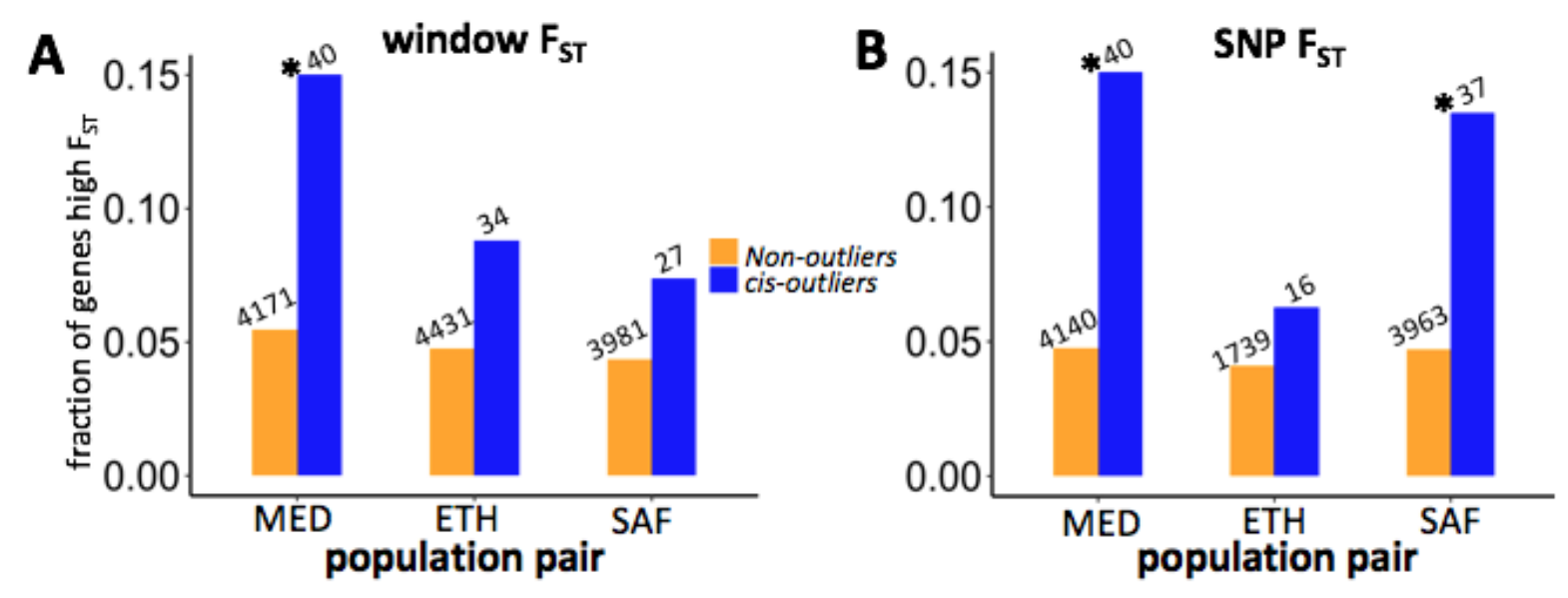


Fig. 7. Enrichment of cis-outliers for genes with high window $F_{S T}(\mathrm{~A})$ and high $\mathrm{SNP} F_{S T}(\mathrm{~B})$ for each population pair. The number above the bar shows the denominator of the fraction. $*$ indicates the fractions are significantly different between cis-effect outliers and non-outliers $(\mathrm{p}<0.05$, Fisher's

819 Exact Test).

821 We further examined potential targets of cis-regulatory local adaptation, excluding genes with cis-

822 effects and trans-effects in the opposite directions. For the cis-genes of the MED pair, Ciaol and

823 Cyp6a22 showed high window $F_{S T}, C G 42565$ showed high $F_{S T \_S N P m a x}$, and CG8034 and Cyp6a17

824 showed both. CG8034 (a predicted monocarboxylic acid transporter) was also cited above as having

825 parallel trans-regulatory evolution between the MED and ETH pairs, and this gene's cis- and trans-

826 effects were both upregulated in FR relative to EG in MED. CG42565 represents a differentially-

827 spliced product of a transcript that alternatively yields an isoform of CG13510, which is upregulated

828 in response to cold (Qin et al. 2005). Interestingly, Cyp6a22 and Cyp6a17 belong to the cytochrome

829 P450 protein family (Cyp6a22 is 248 bp upstream of Cyp6a17). This region harbors a polymorphic

830 deletion of Cyp6a17 which is associated with both colder temperature preference (Kang et al. 2011;

831 Chakraborty et al. 2018) and lower insecticide resistance (Duneau et al. 2018). Based on the

832 diagnostic SNPs for Cyp6a17 (Good et al. 2014), we found the France-enriched allele for Cyp6a22 is

833 likely linked to the Cyp6a17 deletion. Likewise, at the population level, the frequency of intact

834 Cyp6a17 copy is 0.44 in France and 0.95 in Egypt. Hence, adaptive expression differences may

835 sometimes be driven by gene copy number differentiation between populations. For the $c i s$-genes of

836 ETH, RpL24 showed high window $F_{S T}$ and KrT95D showed high $F_{S T-S N P m a x}$. For SAF, GXIVsPLA2

837 showed high $F_{S T_{-} S N P \max }$, while $A G O 2$ showed both high window $F_{S T}$ and high $F_{S T_{-} S N P \max } . A G O 2$ is

838 involved with antiviral defense and developmental regulation (Deshpande et al. 2005; Nayak et al.

839 2010) and was previously found to contain fixed differences between European and African

840 populations (Pool 2015). For the genes showing high $F_{S T \_S N P m a x}$ in any pair, we plotted the SNP $F_{S T}$

841 along the gene region and nearby $20 \mathrm{~kb}$ to show the sites that may be the most likely targets of

842 selection (Fig. 8). For $C G 42565$, $C G 8034$ and $K r T 95 D$, we observed that the highest $F_{S T}$ sites were

843 located within the gene regions. While for GXIVSPLA2, the highest SNP $F_{S T}$ was 1758 bp

844 downstream of the gene. For AGO2, the highest SNP $F_{S T}$ was 6359 bp downstream but the third

845 highest SNP $F_{S T}$ was within the gene. Overall, the genetic differentiations between cold- and warm-

846 derived populations around these candidate genes can be quite local, but the linked signal of natural

847 selection can extend further, and there are often multiple SNPs that could represent plausible targets

848 of local adaptation within and outside a given gene region. 

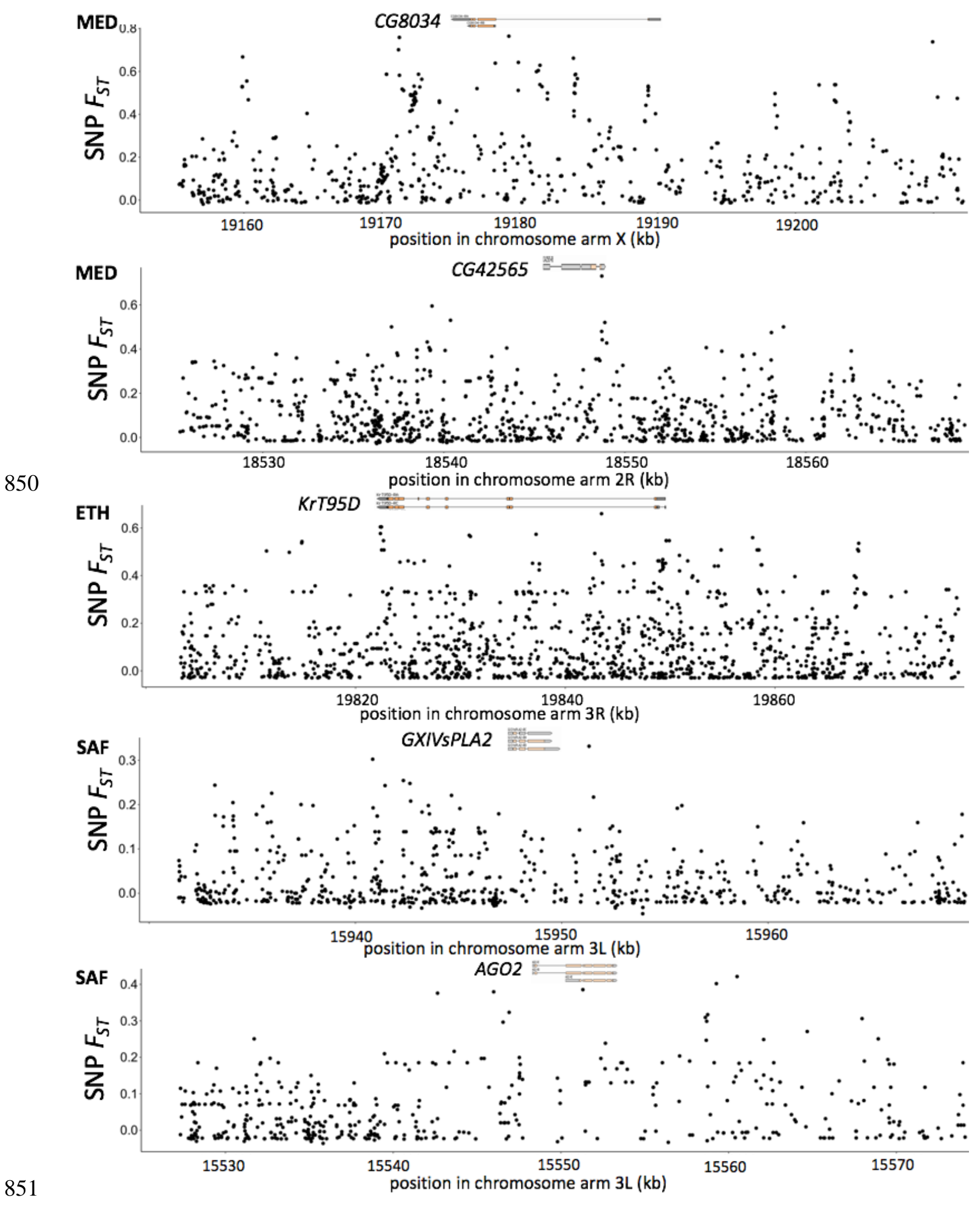
852

Fig 8. Local peaks of $F_{S T}$ between warm- and cold-derived populations are observed at some candidate genes for adaptive cis-regulatory evolution. SNP $F_{S T}$ along the candidate genes with flanking regions of $20 \mathrm{~kb}$ is shown. The top diagram depicts the coding (orange) and non-coding (gray) exon, captured from GBrowse 2 of D. melanogaster (R5.57) from FlyBase (St. Pierre et al. 2014). Cyp6a17 is not plotted because of the known gene-scale polymorphic deletion.

\section{Discussion}

Parallel evolution has often been studied at the population genetic and trait levels, but it has less frequently been analyzed at the transcriptome level (Stern 2013; Juneja et al. 2016). In this study, we used three recent instances of adaptation to colder climates in Drosophila melanogaster to study the evolution of gene expression and alternative splicing. We found a unique pattern of transcriptomic evolution in the high altitude EF sample, involving elevated expression of highly expressed muscle proteins and many other genes. We found the locations of differentially expressed genes on the $\mathrm{X}$ chromosome versus the autosomes varies among developmental stages, with the adult female stage having relatively more differentially expressed X-linked genes than the larval stage. We then saw signals of parallel evolution in expression that were higher for larval and adult stages than for pupa. Further, we studied cis- and trans-regulatory evolution in the context of this ecological adaptation, finding that the relative roles of these regulatory mechanisms differ strongly among population pairs. And we found a signal of trans-regulation contributing more predictably to the parallel evolution between population pairs. Finally, those outliers showing cis-effects were enriched for high genetic differentiation between populations, suggesting that some of them were the direct targets of selection in cold environments.

Previous comparative transcriptomic studies on multiple pairs of Drosophila populations/species have found parallel gene expression differences between high and low latitude populations (Zhao et al. 2015; Juneja et al. 2016). However, because both clines in Australia and North America came from admixtures between European and African ancestry and tropical populations in both clines have a greater proportion of African ancestry (Bergland et al. 2016), it is hard to disentangle adaptive divergence between high and low latitude populations from demographic effects. The demographic influences may be smaller when comparing clines between species pairs (Zhao et al. 2015). Interestingly, the latter study found $10 \%$ to $20 \%$ differential expressed genes between high and low latitude populations were shared and changed co-directionally between two species D. melanogaster 
and $D$. simulans. The percentages are even higher than what we found between population pairs at adult stages (3\% to $10 \%)$.

One important reason for the milder patterns of parallelism among our population pairs may be the different selection agents in their unique habitats. In Zhao et al. 2015, samples from the high and low latitude populations from both species were collected in the same areas. While for us, the coldderived FR population from the MED pair colonized a higher latitude environment than the related warm population, whereas the other two cold-derived populations colonized higher altitude environments where the selection agents may include air pressure, desiccation and ultraviolet radiation (Pool et al. 2017). Although EF and SD have both adapted to higher altitudes (EF at 3,070 meters above sea level, SD at 2,000), SD is seasonally cold (like FR) whereas EF is perpetually cool. We should also note that the standing variation available for adaptation may have differed between our cold-derived populations due to their distinct demographic histories, including the trans-Saharan bottleneck affecting the MED pair and a milder bottleneck in the history of the ETH pair, but no meaningful bottleneck involving the SAF pair (Sprengelmeyer et al. 2020). Hence, although we do

900 significant evidence of parallel gene regulatory adaptation, we suggest that there are both ecological

901 and population genetic reasons to expect substantial non-parallel adaptation as well.

903 Notably, the EF population exhibits distinct phenotypic evolution such as darker pigmentation

904 (Bastide et al. 2014), larger body size (Pitchers et al. 2013; Lack et al. 2016b), and reduced

905 reproductive rate (Lack et al. 2016b). This distinct evolution in the EF population may explain the

906 strong directionality in gene expression changes between EF and EA for the outliers (Fig. 2), the

907 greater abundance of trans-regulatory outliers, and the elevated levels of co-regulation among

908 outliers (Fig. 6). Therefore, the underlying transcriptomic evolution for EF may partly reflect its

909 unique phenotypic evolution, not just adaptation to lower temperature. For example, the upregulation

910 of muscle proteins could reflect the differential abundance of tissues between these Ethiopian

911 populations that differ in size. This size evolution may have also altered the relative proportions of

912 different cell types, which may have driven some of the population differences in gene regulation

913 observed from our whole-organism samples. Future tissue-specific or cell type-specific expression

914 studies involving the EF population can help to examine these possibilities.

916 We found some evidence of parallel expression evolution between our cold-adapted populations.

917 Developmental stage has a strong effect on the levels of this parallelism, with adult and larva 
918 showing significant parallelism while pupa showed a much weaker pattern (Table 1; Figure 4). This

919 is consistent with the observations that larval and adult stages show local adaptation to native

920 temperature but not the pupal stage (Austin and Moehring 2020). It is possible that pupal

921 metamorphosis might reflect a relatively constrained developmental program that limits opportunities

922 for thermal adaptation. The high level of detected parallelism in larvae could also reflect a higher

923 detection power due to less tissue diversity and hence broader spatiotemporal expression of relevant

924 differences. The intriguing pattern of anti-parallelism for some combinations of the pupal stages

925 might suggest that other selective agent is more important than cold (e.g., oxygen level, ultraviolet

926 radiation) for certain population pairs and the direction of selection on gene expression is opposite to

927 the cold. Further, the anti-directional pattern at the pupal stage could be caused by different rates of

928 development for cold-derived populations relative to the warm-derived ones in different pairs.

929 Evidence for such differences is mixed: rates were found to differ between high and low latitude

930 populations in Australia (James \& Partridge 1995) but not between our EF and ZI populations (Lack

931 et al. 2016b). Because tissues at different days can generate a wide range of gene expression

932 difference (Hsu et al. 2019), if the cold-derived population develops faster than the warm-derived

933 one in one pair but in another pair the cold-derived population develops slower than the respective

934 warm-derived one, many of the expression differences will be anti-directional between the two pairs.

935 Moreover, because the pupal and larval samples were mixed sex, different rates of development for

936 males and females could led to a biased sex ratio in a sample, especially for pupae (Testa et al. 2013).

937 If the sex ratio bias happened in the cold-derived population in one pair but the warm-derived

938 population in another, it could conceivably result in anti-directional patterns for sex-biased genes.

940 Compared to the expression abundance, the pattern of parallelism is much weaker for intron usage

941 (Fig S7), which may partly stem from lower power to detect intron usage change (only a small

942 proportion of reads are informative for exon junctions). However, we still found the MED pair and

943 SAF pair show more parallel changes than the combinations with the ETH pair, which is consistent

944 with results for expression abundance. Given the increasing evidence for alternative splicing

945 contributing to environmental response and adaptation (e.g., Singh et al. 2017; Signor and Nuzhdin

946 2018; Smith et al. 2018), we need to study both expression abundance and splicing to fully

947 understand the evolution at the transcriptome level. The development of sequencing approaches with

948 long reads that cover the entire transcripts (e.g., Iso-Seq) will enable us to quantify isoforms

949 frequency directly and broaden the scope of alternative splicing variation that can readily be

950 quantified. Since splicing changes during development and among tissues (Brown et al. 2014; 
951 Gibilisco et al. 2016), a detailed sampling throughout development of different tissues will also be

952 necessary to understand the role of splicing on ecological adaptation.

954 We found trans-effects on expression were more common than the cis-effects across the

955 transcriptome (Table 3), which is consistent with some previous studies (e.g., McManus et al. 2010;

956 Coolon et al. 2014; Albert et al. 2018; Glaser-Shmitt et al. 2018) but not with others (e.g., Lemmon

957 et al. 2014; Mack et al. 2016). The transcriptome-wide prevalence of trans-effects may be caused by

958 random regulatory changes biased toward trans-regulation because of the larger trans-mutational

959 target size (Landry et al. 2007; Metzger et al. 2016). Or, trans-regulatory changes may have higher

960 potential for coordinate regulation of multiple genes in networks (Metzger et al. 2016; Liu et al.

961 2019). To focus on the evolved changes potentially related to adaptation, we compared the

962 proportion of genes with cis-/trans-effects for $P_{S T}$ outliers and to those for non-outliers and saw both

963 effects were enriched in outliers in certain pairs (Fig. 6A \& B). These results indicate that the

964 mechanisms of adaptive gene regulatory evolution are highly population-specific, and that either

965 regulatory mechanism has the potential to play a disproportionate role in ecological adaptation.

967 Moreover, we found a predominance of trans-effects associated with parallel outliers than the non-

968 parallel outliers (Fig. 6E \& F). In part because of the larger mutational target size of trans-regulatory

969 variation for a given target gene, the standing genetic variation for trans-regulatory variants may be

970 higher than the cis-ones in the ancestral population and therefore the trans variants can respond to

971 selection in different population pairs. However, studies in Arabidopsis thaliana and Capsella

972 grandiflora find that trans-eQTLs tend to have lower minor allele frequencies than cis-eQTLs

973 (Zhang et al. 2011; Josephs et al. 2020) but it is unclear whether these populations represent the

974 ancestral state before experiencing environmental changes. Also, the potential capacity of trans-

975 regulatory factors to co-regulate many genes may amplify the probability of parallel changes between

976 population pairs. Furthermore, since we used whole-body adult samples, it is possible that some

977 trans-acting factors regulated genes similarly across tissues while the some cis-effects were tissue-

978 specific and were undetected in our mixed-tissue samples. Finally, we emphasize that our study

979 focuses on regulatory changes that may have relatively larger effects (in focusing on $P_{S T}$ outliers, and

980 in basing cis/trans analysis on strains showing clearer differences); but small changes may be

981 important for regulatory evolution as well, and may be differentially represented between categories

982 (e.g. cis vs. trans, parallel vs. non-parallel). 
984 When we considered genes/introns showing both cis- and trans-effects, we observed that the two

985 types of effects were generally in opposite directions (anti-directional; Table 3). This is consistent

986 with the idea that gene regulation is under stabilizing selection in general and gene regulatory

987 networks evolve negative feedback to buffer effects of regulatory changes (Denby et al. 2012;

988 Coolon et al. 2014; Bader et al. 2015; Fear et al. 2016). With regard to our $P_{S T}$ outliers, it is possible

989 that cis-acting changes might have evolved to compensate for unfavorable pleiotropic impacts of

990 adaptive trans-regulatory evolution (or possibly vice-versa). However, negative correlations between

991 cis- and trans-effects can also be an artifact coming from the measurement error on F1 expression

992 data. Because the F1 data was used to estimate ASE and compared it to 0.5 (cis-effect null) and to

993 parental expression proportion (trans-effect null), measurement error will introduce artifactual

994 negative correlation between cis- and trans-acting changes. Therefore, whether the opposing effects

995 between cis- and trans-acting changes are biologically meaningful will require further study. As

996 Fraser (2019) and Zhang and Emerson (2019) proposed, using independent F1 replicates or other

997 approaches such as eQTL mapping to infer cis- and trans-effects separately is necessary to affirm

998 evidence of compensatory evolution.

1000 We expect that the adaptive expression divergence caused by cis-regulatory changes should leave a

1001 signal in the genetic variation of the nearby genomic region. Therefore, we used $F_{S T}$ statistics to

1002 quantify genetic differentiation for the region around the focal genes. Window $F_{S T}$ is sensitive to

1003 classic hard sweeps, and relatively useful for incomplete sweeps and moderately soft sweeps, but it is

1004 less useful for soft sweeps with higher initial frequencies of the beneficial allele (Lange and Pool

1005 2016), for which SNP $F_{S T}$ may be more sensitive. Indeed, a previous genomic study on these same

1006 populations found a stronger signal of parallel change for SNP $F_{S T}$ than for window $F_{S T}$ genome-wide

1007 (Pool et al. 2017). Here, we found genes with outlier cis-effects are enriched for those that show high

$1008 F_{S T}$, especially those with high SNP $F_{S T}$ (Fig. 7A \& B). Hence, standing genetic variants may have

1009 contributed importantly to the cis-regulatory changes for adaptation in our populations. Genes with

1010 both significant $c i s$-effects and high $F_{S T}$ are likely to be the direct targets of the environmental

1011 selection and good candidate for future mechanistic studies.

1012

1013 Using three natural fly population pairs with recent adaptive divergence, our study found intriguing

1014 patterns of parallel evolution in gene expression and provided new insights on the underlying

1015 regulatory effect. In the future, using other approaches to study cis-and trans-effects in these 
1016 populations would be necessary, such as eQTL mapping, which can provide more genetic

1017 information about the trans-regulatory loci. Also, studying the gene expression in different tissues,

1018 including different organs from males and females, would provide us a clearer and more

1019 comprehensive picture about parallel gene expression evolution. It would also be informative to

1020 study other phenotypes besides gene expression that are more related to thermotolerance such as the

1021 metabolic pathways identified in this study or nervous system function related to chill coma.

1022 Moreover, studying the phenotypic plasticity at different developmental stages could help to explain

1023 the different patterns of parallelism in expression evolution across stages and allow us to better

1024 understand the importance of local adaptation versus plasticity in thermotolerance.

\section{Data Availability}

1029

The raw RNAseq reads are available from the Sequence Read Archive (SRA) under accessions

\section{Acknowledgements}

1038

We thank Colin Dewey for helpful discussions and the UW-Madison Center for High Throughput Computing (CHTC) for cluster usage. We thank Jeremy Lange for suggestions on Cyp6a17 gene analysis. This work was funded by NSF DEB grant 1754745 to JEP and by NIH NIGMS grant F32GM106594 to JBL. from the Drosophila Genome Nexus (Lack et al. 2016; http://www.johnpool.net/genomes.html). Custom R and Perl scripts for the cis- and trans- effects simulation and analysis can be found at https://github.com/YuhengHuang87/simulation cis trans.

\section{References}

Adrion JR , Hahn MW , Cooper BS. 2015. Revisiting classic clines in Drosophila melanogaster in the age of genomics. Trends Genet 8:434-444.

Alasoo K , Rodrigues J , Danesh J , Freitag DF , Paul DS , et al. 2019. Genetic effects on promoter usage are highly context-specific and contribute to complex traits. Elife. 8 . doi:10.7554/eLife.41673

1053

Albert FW, Bloom JS, Siegel J, Day L, Kruglyak L. 2017. Genetics of trans-regulatory variation in gene expression. eLife 100:371-44.

Arthaud L, Ben Rokia-Mille S, Raad H, Dombrovsky A, Prevost N, et al. 2011. Trade-off between toxicity and signal detection orchestrated by frequency- and density-dependent genes. PLoS One 6: e19805. 
1054

1055

1056

1057

1058

1059

1060

1061

1062

1063

1064

1065

1066

1067

1068

1069

1070

1071

1072

1073

1074

1075

1076

1077

1078

1079

1080

1081

1082

1083

1084

1085

1086

1087

1088

Austin CJ, Moehring AJ. 2019. Local thermal adaptation detected during multiple life stages across populations of Drosophila melanogaster. J. Evol. Biol. 32, 1342-1351.

Bader DM, Wilkening S, Lin SG, Tekkedil MM, Dietrich K, et al. 2015. Negative feedback buffers effects of regulatory variants. Mol Syst Biol 11:785.

Barbosa-Morais NL, Irimia M, Pan Q, Xiong HY, Gueroussov S, et al. 2012. The evolutionary landscape of alternative splicing in vertebrate species. Science 338:1587-93.

Bastide H, Yassin A, Johanning EJ, Pool JE. 2014. Pigmentation in Drosophila melanogaster reaches its maximum in Ethiopia and correlates most strongly with ultra-violet radiation in sub-Saharan Africa. BMC Evol Biol 14:222-14.

Benjamini Y, Hochberg Y. 1995. Controlling the false discovery rate-a practical and powerful approach to multiple testing. J Royal Stat Soc B 57:289-300.

Bergland AO, Tobler R, González J, Schmidt P, Petrov D. 2016. Secondary contact and local adaptation contribute to genome-wide patterns of clinal variation in Drosophila melanogaster. Mol Ecol 25:1157-1174.

Božičević V, Hutter S, Stephan W, Wollstein A. 2016. Population genetic evidence for cold adaptation in European Drosophila melanogaster populations. Mol Ecol 25:1175-1191.

Brommer JE. 2011. Whither $P_{S T}$ ? The approximation of $Q_{S T}$ by $P_{S T}$ in evolutionary and conservation biology. J Evol Biol 24:1160-1168.

Brown JB, Boley N, Eisman R, May GE, Stoiber MH, Duff MO, et al. 2014. Diversity and dynamics of the Drosophila transcriptome. Nature. 7515:393-9.

Cao W, Edery I. 2017. Mid-day siesta in natural populations of D. melanogaster from Africa exhibits an altitudinal cline and is regulated by splicing of a thermosensitive intron in the period clock gene. BMC Evol. Biol. 17: 32.

Catalan A, Hutter S, Parsch J. 2012. Population and sex differences in Drosophila melanogaster brain gene expression. BMC Genomics 13:1-12.

Chakraborty M, VanKuren NW, Zhao R, Zhang X, Kalsow S, Emerson JJ. 2017. Hidden genetic variation shapes the structure of functional elements in Drosophila. Nat Genet 50:20-25.

Chen J, Nolte V, Schlötterer C. 2015. Temperature Stress Mediates Decanalization and Dominance of Gene Expression in Drosophila melanogaster. PLoS Genet 11:e1004883.

Comeron JM, Ratnappan R, Bailin S. 2012. The many landscapes of recombination in Drosophila melanogaster. PLoS Genet 8:e1002905.

Conte GL, Arnegard ME, Peichel CL, Schluter D. 2012. The probability of genetic parallelism and convergence in natural populations. Proc Royal Soc B 279:5039-5047.

Coolon JD, McManus CJ, Stevenson KR, Graveley BR, Wittkopp PJ. 2014. Tempo and mode of regulatory evolution in Drosophila. Genome Res 24:797-808. 
Cowles CR, Hirschhorn JN, Altshuler D, Lander ES. 2002. Detection of regulatory variation in mouse genes. Nat Genet 32:432-437

Degner JF, Marioni JC, Pai AA, Pickrell JK, Nkadori E, Gilad Y, Pritchard JK. 2009. Effect of read-mapping biases on detecting allele-specific expression from RNA-sequencing data. Bioinformatics 25:3207-3212.

Denby CM, Im JH, Yu RC, Pesce CG, Brem RB. 2012. Negative feedback confers mutational robustness in yeast transcription factor regulation. Proc Natl Acad Sci USA 109:3874-3878.

Deshpande, G., Calhoun, G., Schedl, P. 2005. Drosophila argonaute-2 is required early in embryogenesis for the assembly of centric/centromeric heterochromatin, nuclear division, nuclear migration, and germ-cell formation. Genes Dev. 19:1680-1685.

Dobin A, Davis CA, Schlesinger F, Drenkow J, Zaleski C, Jha S, Batut P, Chaisson M, Gingeras TR. 2013. STAR: ultrafast universal RNA-seq aligner. Bioinformatics 29:15-21.

Duneau D, Sun H, Revah J, San Miguel K, Kunerth HD, Caldas IV, Messer PW, Scott JG, Buchon N. 2018. Signatures of insecticide selection in the genome of Drosophila melanogaster. G3 (Bethesda) 8:3469-3480.

Emerson JJ. 2019. Inferring compensatory evolution of cis- and trans-regulatory variation. Trends Genet 35:1-3.

Fabian DK, Kapun M, Nolte V, Kofler R, Schmidt PS, Schlötterer C, Flatt T. 2012. Genomewide patterns of latitudinal differentiation among populations of Drosophila melanogaster from North America. Mol Ecol 21:4748-4769.

Fear JM, Leon-Novelo LG, Morse AM, Gerken AR, Van Lehmann K, Tower J, Nuzhdin SV, McIntyre LM. 2016. Buffering of genetic regulatory networks in Drosophila melanogaster. Genetics 203:1177-1190.

Fraser HB. 2019. Improving estimates of compensatory cis-trans regulatory divergence. Trends Genet 35:3-5.

Gamazon ER, Stranger BE. 2014. Genomics of alternative splicing: evolution, development and pathophysiology Hum Genet 133:679-687

Gibilisco L, Zhou Q, Mahajan S, Bachtrog D. 2016. Alternative splicing within and between Drosophila species, sexes, tissues, and developmental stages. PLoS Genet 12: e1006464.

Gibson JR, Chippindale AK, Rice AM. 2002. The X chromosome is a hot spot for sexually antagonistic fitness variation. Proc. Biol. Sci. 269: 499-505.

Glaser-Schmitt A, Zecic A, Parsch J. 2018. Gene regulatory variation in Drosophila melanogaster renal tissue. Genetics 210:287-301. 
Hanson D, Hu J, Hendry AP, Barrett RDH. 2017. Heritable gene expression differences between lake and stream stickleback include both parallel and antiparallel components. Heredity 119:339-348.

Hart JC, Ellis NA, Eisen MB, Miller CT. 2018. Convergent evolution of gene expression in two high-toothed stickleback populations. PLoS Genetics 6:e1007443.

Helfrich-Forster, C. 2018. Sleep in Insects. Annu Rev Entomol 63:69-86.

Hoffmann AA, Hallas RJ, Dean JA, Schiffer M. 2003. Low potential for climatic stress adaptation in a rainforest Drosophila species. Science 301:100-102.

Hoffmann AA, Weeks AR. 2007. Climatic selection on genes and traits after a 100 year-old invasion: a critical look at the temper-ate-tropical clines in Drosophila melanogaster from eastern Australia. Genetica 129:133-147.

Hsu SK, Jaksic AM, Nolte V, Barghi N, Mallard F, et al. 2019. A 24 h age difference causes twice as much gene expression divergence as 100 generations of adaptation to a novel environment. Genes 10:89.

Huylmans AK, Parsch J. 2014. Population- and sex-biased gene expression in the excretion organs of Drosophila melanogaster. G3 (Bethesda). 4:2307-15.

Izquierdo JI. 1991. How does Drosophila melanogaster overwinter? Entomol. Exp. Appl. 59: $51-58$.

Innocenti P, Morrow EH. 2010. The sexually antagonistic genes ofDrosophila melanogaster. PLoS Biol. 8: e1000335.

James AC, Partridge L. 1995. Thermal evolution of rate of larval development in Drosophila melanogaster in laboratory and field populations. J. Evol. Biol. 8: 315-330.

Jones FC, Grabherr MG, Chan YF, Russell P, Mauceli E, et al. 2012. The genomic basis of adaptive evolution in threespine sticklebacks. Nature 484:55-61.

Josephs, EB., Lee. YW, Wood CW, Schoen DJ, Wright SI, et al. 2020. The Evolutionary Forces Shaping Cis- and Trans-Regulation of Gene Expression within a Population of Outcrossing Plants. Molecular Biology and Evolution, 37: 2386-2393

Juneja P, Quinn A, Jiggins FM. 2016. Latitudinal clines in gene expression and cis-regulatory element variation in Drosophila melanogaster. BMC Genomics:1-11.

Kang J, Kim J, Choi K-W. 2011. Novel cytochrome P450, cyp6a17, is required for temperature preference behavior in Drosophila. PLoS ONE 6:e29800. 
Kolaczkowski B, Kern AD, Holloway AK, Begun DJ. 2011. Genomic differentiation between temperate and tropical Australian populations of Drosophila melanogaster. Genetics 187:245-260.

Kurmangaliyev YZ, Favorov,AV, Osman NM, Lehmann K-V, Campo D, et al. 2015. Natural variation of gene models in Drosophila melanogaster. BMC Genomics 16:198

Lack JB, Lange JD, Tang AD, Corbett-Detig RB, Pool JE. 2016a. A thousand fly genomes: An expanded Drosophila genome nexus. Mol Biol Evol 33:3308-3313.

Lack JB, Monette MJ, Johanning EJ, Sprengelmeyer QD, Pool JE. 2016b. Decanalization of wing development accompanied the evolution of large wings in high-altitude Drosophila. Proc Natl Acad Sci USA 113:1014-1019.

Lai Z, Kane NC, Zou Y, Rieseberg LH. 2008. Natural variation in gene expression between wild and weedy populations of Helianthus annuus. Genetics 179:1881-1890.

Landry CR, Lemos B, Rifkin SA, Dickinson WJ, Hartl DL. 2007. Genetic properties influencing the evolvability of gene expression. Science 317:118-121.

Lange JD, Pool JE. 2016. A haplotype method detects diverse scenarios of local adaptation from genomic sequence variation. Mole Ecol 25:3081-3100.

Lavington, E., and A. D. Kern, 2017 The effect of common inver-sion polymorphisms In(2L)t and In(3R)Mo on patterns of tran-scriptional variation in Drosophila melanogaster. G3 (Bethesda)7: 3659-3668.

Leder EH, McCairns RJS, Leinonen T, et al. 2015. The evolution and adaptive potential of transcriptional variation in sticklebacks - signatures of selection and widespread heritability. Mol Biol and Evol 32:674-689.

Leinonen T, McCairns RJS, O'Hara RB, Merilä J. 2013. $Q_{S T}-F_{S T}$ comparisons: evolutionary and ecological insights from genomic heterogeneity. Nat Rev Genet. 14:179-190.

Lemmon ZH, Bukowski R, Sun Q, Doebley JF. 2014. The role of cis regulatory evolution in maize domestication. PLoS Genet 10:e1004745-15.

Li B \& Dewey Colin N. 2011. RSEM: accurate transcript quantification from RNA-Seq data with or without a reference genome. BMC Bioinform 12:323

Li H, Handsaker B, Wysoker A, Fennell T, Ruan J, et al. 2009. The Sequence alignment/map (SAM) format and SAMtools. Bioinformatics 25:2078-2079. quantification of RNA splicing using LeafCutter. Nat Genet 50:151-158. Omnigenic Inheritance. Cell 177: 1022-1034.e6.

Losos JB. 2011. Convergence, adaptation, and constraint. Evolution 65:1827-1840. 
Machado HE, Bergland AO, O'Brien KR, Behrman EL, Schmidt PS, Petrov DA. 2016. Comparative population genomics of latitudinal variation in Drosophila simulans and Drosophila melanogaster. Mol Ecol 25:723-740.

Mack KL, Campbell P, Nachman MW. 2016. Gene regulation and speciation in house mice. Genome Res 26:451-461.

Massouras A, Waszak SM, Albarca-Aguilera M, Hens K, Holcombe W, et al. 2012. Genomic Variation and Its Impact on Gene Expression in Drosophila melanogaster. PLoS Genet 8:e1003055.

Mateo L, Ullastres A, González J. 2014. A transposable element insertion confers xenobiotic resistance in Drosophila. PLoS Genet 10:e1004560.

McGirr JA, Martin CH. 2018. Parallel evolution of gene expression between trophic specialists despite divergent genotypes and morphologies. Evol Lett 2:62-75.

McManus CJ, Coolon JD, Duff MO, Eipper-Mains J, Graveley BR, Wittkopp PJ. 2010. Regulatory divergence in Drosophila revealed by mRNA-seq. Genome Res 20:816-825.

McManus CJ, Coolon JD, Eipper-Mains J, Wittkopp PJ, Graveley BR. 2014. Evolution of splicing regulatory networks in Drosophila. Genome Res 24:786-796.

Meiklejohn CD, Coolon JD, Hartl DL, Wittkopp PJ. 2014. The roles of cis- and trans-regulation in the evolution of regulatory incompatibilities and sexually dimorphic gene expression. Genome Res 24:84-95.

Merilä J. 1997. Quantitative trait and allozyme divergence in the greenfinch (Carduelis chloris, Aves: Fringillidae). Biol J Linn Soc 61:243-266.

Metzger BP, Duveau F, Yuan DC, Tryban S, Yang B, et al., 2016 Contrasting frequencies and effects of cis- and trans-reg-ulatory mutations affecting gene expression. Mol. Biol. Evol. 33:1131-1146.

Müller L, Hutter S, Stamboliyska R, Saminadin-Peter SS, Stephanet W, et al. 2011. Population transcriptomics of Drosophila melanogaster females. BMC Genomics 12: 81.

Nagoshi E, Sugino K, Kula E, Okazaki E, Tachibana T, et al. 2010 Dissecting differential gene expression within the circadian neuronal circuit of Drosophila. Nat. Neurosci. 13: 60-68.

Nandamuri SP, Conte MA, Carleton KL. 2018. Multiple trans QTL and one cis-regulatory deletion are associated with the differential expression of cone opsins in African cichlids. BMC Genomics. 19:945. antagonizes Argonaute 2 to modulate antiviral defense in Drosophila. Nat. Struct. Mol. Biol. 17: 547-554.

Osada N, Miyagi R, Takahashi A. 2017. Cis- and trans-regulatory effects on gene expression in a natural population of Drosophila melanogaster. Genetics 206:2139-2148. 
Pitchers W, Pool JE, Dworkin I. 2012. Altitudinal clinal variation in wing size and shape in african Drosophila melanogaster: one cline or many? Evolution 67:438-452.

Pool JE, Braun DT, Lack JB. 2016. Parallel evolution of cold tolerance within Drosophila melanogaster. Mol Biol Evol 34:349-360.

Pool JE, Corbett-Detig RB, Sugino RP, Stevens KA, Cardeno CM, Crepeau MW, Duchen P, Emerson JJ, Saelao P, Begun DJ, et al. 2012. Population genomics of sub-Saharan Drosophila melanogaster: African diversity and non-African admixture. PLoS Genet 8:e1003080-24.

Pool JE, Nielsen R. 2007 Population size changes reshape genomic patterns of diversity. Evolution 61:3001-3006.

Pool JE. 2015. The Mosaic Ancestry of the Drosophila Genetic Reference Panel and the $D$. melanogaster reference genome reveals a network of epistatic fitness interactions. Mol Biol Evol 32:3236-3251.

Powell JR. 1997. Progress and prospects in evolutionary biology, The Drosophila model. New York, NY: Oxford University Press.

Qin, W., S. J. Neal, R. M. Robertson, J. T. Westwood, and V. K. Walker. 2005. Cold hardening and transcriptional change in Drosophila melanogaster. Insect Mol. Biol. 14:607-613.

Reinhardt JA, Kolaczkowski B, Jones CD, Begun DJ, Kern AD. 2014. Parallel geographic variation in Drosophila melanogaster. Genetics 197:361-373.

Rosenblum EB, Parent CE, Brandt EE. 2014. The molecular basis of phenotypic convergence. Annu Rev Ecol Evol Syst 45:203-226.

Sackton TB, et al. 2019. Convergent regulatory evolution and loss of flight in paleognathous birds. Science 364:74-78.

Said I, Byrne A, Serrano V, Cardeno C, Vollmers C, and Corbett-Detig R. 2018. Linked genetic variation and not genome structure causes widespread differential expression associated with chromosomal inversions. Proceedings of the National Academy of Sciences 115:5492-5497. expression level and selective constraints on trans- and cis-regulatory changes in yeast. Mol Biol Evol 30:2121-2133.

Schluter D. 2000. The ecology of adaptive radiation. Oxford Univ. Press, New York. populations of Drosophila melanogaster. Evolution 62:1204-1215. measurement of allele-specific transcripts differing by a single nucleotide. PCR Methods Appl.1: 160-163. 
Signor S, Nuzhdin S. 2018. Dynamic changes in gene expression and alternative splicing mediate the response to acute alcohol exposure in Drosophila melanogaster. Heredity 121:342-360.

Singh P, Börger C, More H, Sturmbauer C. 2017. The role of alternative splicing and differential gene expression in cichlid adaptive radiation. Genome Biol Evol 9:2764-2781.

Smith CCR, Tittes S, Mendieta JP, Collier-Zans E, Rowe HC, Rieseberg LH, Kane NC. 2018. Genetics of alternative splicing evolution during sunflower domestication. Proc Natl Acad Sci USA 115:6768-6773.

Sokolowski, MB, Bauer SJ, Wai-Ping V, Rodriguez L, Wong JL, Kent C.1986. Ecological genetics and behavior of Drosophila melanogaster larvae in nature. Animal Behavior 34:403-408.

Sprengelmeyer QD, Mansourian S, Lange JD, Matute DR, Cooper BS, Jirle EV, Stensmyr MC, Pool JE. 2018. Discovery of Drosophila melanogaster from Wild African Environments and Genomic Insights into Species History. biorxiv 45:1153-13.

St Pierre SE, Ponting L, Stefancsik R, McQuilton P, the FlyBase Consortium. 2013. FlyBase 102—advanced approaches to interrogating FlyBase. Nucleic Acids Res 42:D780-D788.

Stern DL. 2013. The genetic causes of convergent evolution. Nat Rev Genet 14:751-764.

Stevenson KR, Coolon JD, Wittkopp PJ. 2013. Sources of bias in measures of allele-specific expression derived from RNA-seq data aligned to a single reference genome. BMC Genomics 14:536.

Svetec N, Zhao L, Saelao P, Chiu JC, and Begun DJ. 2015. Evidence that natural selection maintains genetic variation for sleep in Drosophila melanogaster. BMC Evol. Biol. 15: 41.

Testa ND, Ghosh SM, and Shingleton AW. 2013. Sex-specific weight loss mediates sexual size dimorphism in Drosophila melanogaster. PLoS One 8:e58936.

Thurmond J, Goodman JL, Strelets VB, Attrill H, Gramates LS, et al. 2019. FlyBase 2.0: the next generation. Nucleic Acids Res. 47:D759-D765. doi:10.1093/nar/gky1003.

Venables JP, Tazi J, Juge F. 2011. Regulated functional alternative splicing in Drosophila. Nucleic Acids Res 40:1-10.

Verta JP, Jones FC. 2019. Predominance of cis-regulatory changes in parallel expression divergence of sticklebacks. eLife. 8:e43785.

Vicoso, B, and B. Charlesworth, 2006 Evolution on the X chromosome: unusual patterns and processes. Nat. Rev. Genet. 7: 645-653. signature of regulatory cold adaptation in temperate Drosophila melanogaster. BMC Genomics, 17:574. 
Wang D, Sung HM, Wang TY, Huang CJ, Yang P, et al. 2007. Expression evolution in yeast genes of single-input modules is mainly due to changes in trans-acting factors. Genome Research 17: 1161-1169. https://doi.org/10.1101/gr.6328907

Wei Y, Reveal B, Reich J, Laursen WJ, Senger S, et al. 2014. TORC1 regulators Iml1/GATOR1 and GATOR2 control meiotic entry and oocyte development in Drosophila. Proc. Natl. Acad. Sci. U.S.A. 111: E5670--E5677.

Wittkopp PJ, Kalay G. 2011. Cis-regulatory elements: molecular mechanisms and evolutionary processes underlying divergence. Nat Rev Genet 13:59-69.

Wittkopp PJ, Haerum BK, Clark AG. 2004. Evolutionary changes in cis and trans gene regulation. Nature 430:85-88.

Wittkopp PJ, Haerum BK, Clark AG. 2008. Regulatory changes underlying expression differences within and between Drosophila species. Nat Genet 403:346-350.

Yan H, Yuan W, Velculescu VE, Vogelstein B, Kinzler KW. 2002. Allelic variation in human gene expression. Science 297:1143

Yu G, Wang L, Han Y, He Q. 2012. clusterProfiler: an R package for comparing biological themes among gene clusters. OMICS 16:284-287.

Yvert G, Brem RB, Whittle J, Akey JM, Foss E, et al. 2003. Trans-acting regulatory variation in Saccharomyces cerevisiae and the role of transcription factors. Nat. Genet. 35:57-64.

Zhao L, Begun DJ. 2017. Genomics of parallel adaptation at two timescales in Drosophila. PLoS Genet 13:e1007016.

Zhao L, Wit J, Svetec N, Begun DJ. 2015. Parallel gene expression differences between low and high latitude populations of Drosophila melanogaster and D. simulans. PLoS Genet 11:e1005184. Arabidopsis thaliana. Genome Res. 21:725-733.

Zhang Y, Wang ZH, Liu Y, Chen Y, Sun N, et al. 2019. PINK1 Inhibits Local Protein Synthes is to Limit Transmission of Deleterious Mitochondrial DNA Mutations. Mol Cell:1127-1137. 